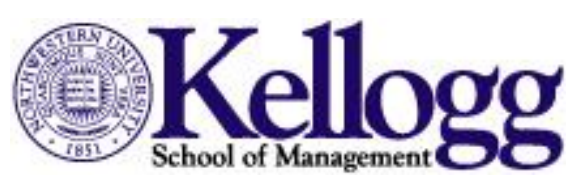

CMSEMS

Center for Mathematical Studies in Economics

And Management Science

Disc ussion Paper \#1543

Ambiguity in Dynamic Contracts

Martin Szydlowski

Northwestem University

January 16, 2012

J EL Classification: D82, D86, M52

Keywords: Dyna mic contract, principal-agent model, a mbiguity a version, continuous time
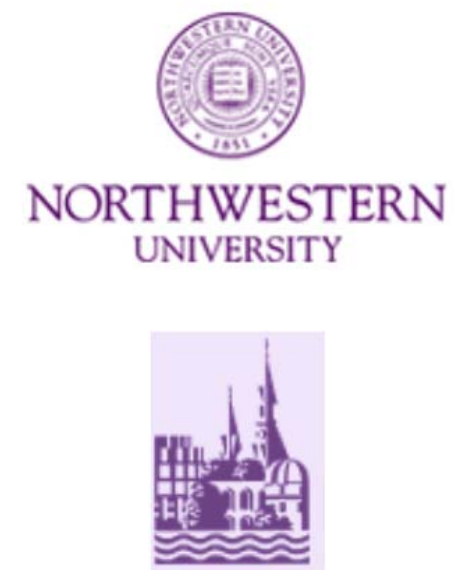


\title{
Ambiguity in Dynamic Contracts*
}

\author{
Martin Szydlowski ${ }^{\dagger}$
}

January 16, 2012

\begin{abstract}
I study a dynamic principal agent model in which the effort cost of the agent is unknown to the principal. The principal is ambiguity averse, and designs a contract which is robust to the worst case effort cost process. Ambiguity divides the contract into two regions. After sufficiently high performance, the agent reaches the over-compensation region, where he receives excessive benefits compared to the contract without ambiguity, while after low performance, he enters the under-compensation region. Ambiguity also causes a disconnect between the current effort cost and the strength of incentives. That is, even when the agent is under-compensated, his incentives are as strong as in the over-compensation region, since the principal fears the agent might shirk otherwise.

Under ambiguity, the agent's true effort cost does not need to equal the worst-case. I analyze the agent's incentives for this case, and show that the possibility of firing is detrimental to the agent's incentives. I study several extensions concerning the timing structure and the nature of the principal's ambiguity aversion.
\end{abstract}

\section{Introduction}

Modern firms operate in a business environment which is subject to unexpected changes, such as the entry of competitors, arrivals of new technologies, new legislation, or variations in customer demands. While firms need to motivate their major employees, shocks to the

\footnotetext{
*I would like to thank Eddie Dekel, Daniel Garrett, Wojchiech Olszewski and Marciano Siniscalchi for helpful discussions, and Jeff Ely, Mike Fishman and Bruno Strulovici for their guidance.

${ }^{\dagger}$ Northwestern University, Department of Economics, 302 Arthur Andersen Hall, 2001 Sheridan Road Evanston, IL 60208-2600. Email: martin.szydlowski@u.northwestern.edu

JEL Classification: D82, D86, M52
} 
environment make this task difficult. Many such changes are not anticipated at the time an employment contract is written, and their impact on the manager's individual productivity or the difficulty of his day-to-day activities is impossible to verify and to communicate.

In this paper, I study a dynamic contracting problem in which the firm does not know the arrival nor the impact of shocks to the environment. I model the firm's lack of knowledge as ambiguity aversion. That is, the firm is aware that it does not know the probability law of the shock process, and it offers the manager an incentive contract which seeks to maximize the firm's payoff and provide incentives to the agent under the worst-case realization. The contribution of my paper is to characterize which impact the firm's lack of knowledge has on the shape of the optimal contract, and to provide a the dynamics of the worst-case process. My main example is when the ambiguity is about the manager's effort cost. For instance, keeping effort constant, the difficulty of the manager's work could vary depending on the competitive pressure faced by the firm, or the manager's perception of the difficulty of the work could change over time, even though his responsibilities stay the same.

In this case, the optimal contract has several novel features, which are driven by the dynamics of the worst-case process. First, the contract space is divided into an over- and an under-compensation region. After sufficiently high performance of the manager, the worst-case dictates that the manager's disutility from effort is at the highest possible level. Consequently, the firm over-estimates the effort cost of the manager, and compensates him too much compared to the case without ambiguity. Similarly, after low performance, the worst-case implies that the manager's effort cost is low, and he will be under-compensated.

Hence, my paper offers a new explanation for why managers may be overcompensated, a question which has received significant attention in both the popular press and academic literature, see e.g. Bebchuk and Fried (2006) and Gabaix and Landier (2008). Here, overcompensation does not occur because of managerial power, or the exploitation of information rents, but because of the firm's desire to insure against the worst-case process.

I find that both the over- and under-compensation regions are persistent. Once the manager is in the over-compensation region, he will stay there in expectation, and only drop to the under-compensation region after a history of very low performance. In the undercompensation region, the manager will face a higher firing probability. Although he will 'move up' in expectation, the speed at which this advancement occurs is lower, and so is the probability of attaining the over-compensation region before being fired, compared to the case without ambiguity.

Ambiguity also introduces a disconnect between the manager's pay-performance sensitivity 
and his current effort cost. In a dynamic contract without ambiguity, the agent's expected future utility reacts optimally to the noise in the output process, and at each point in time, the strength of this reaction is proportional to the agent's current effort cost. In my setting, the firm is forced to set the pay-performance sensitivity at the highest level at all times, since otherwise the worst-case process would induce the agent to shirk. Thus, the manager is over-incentivized no matter whether he is over- or under-compensated. This feature of my model offers an alternative answer to the puzzle raised by Murphy (2003), who find that many employees below the CEO level receive stock options, even though their individual impact on firm performance does not seem to be sufficiently large to warrant such strong incentives.

When the principal is ambiguity averse, the worst-case effort cost process does not have to be the agent's actual one. Instead, we can think of the firm as misjudging the agent's effort cost. In Section 5, I study how an agent with a constant effort cost responds to such a misspecified incentive contract. Contrary to what has been found in the contract theory literature, ${ }^{1}$ the possibility of firing does not always serve as a motivator for the agent, and in fact can induce the agent to shirk. The reason for this is the following. The agent's marginal utility of effort depends on the payments he is promised to collect in the future in exchange for exerting effort. However, when the firing probability is high, the agent has a low chance of collecting his promised payments before he is laid off, and thus his marginal utility of effort will diminish. If the effort cost is sufficiently high, the agent will then shirk. In some cases, this implies that instead of expecting to move up to the over-compensation region in expectation, the agent will drift towards firing, unless he is lucky and displays good performance even though he does not work.

In line with this intuition, agents who shirk will always be risk-loving, at least for the time that they are shirking, while agents who work are always risk averse. The latter can expect enter and then to stay in the over-compensation region forever if the noise in the output process is sufficiently small, and thus they will always prefer to bear less risk.

I study several extensions of this framework. In Section 4.3, I show that the optimal contract can be implemented using an equity share, and performance sensitive debt. In line with the result on the pay-performance sensitivity, the equity share is higher than in the case without ambiguity, while the performance sensitive debt is used to adjust the firm's cash flows in the over- and under-compensation regions. The principal's decision problem under ambiguity

\footnotetext{
${ }^{1}$ Theoretical works which argue that firing provides incentives to the agent are numerous, and include Spear and Wang (2005), Wang (2010), Garrett and Pavan (2010b), Fong and Li (2010), as well as a number of works in inspired by Sannikov (2008), such as Biais et al. (2010), DeMarzo and Sannikov (2006), DeMarzo et al. (2010) and He (2009).
} 
can be alternatively understood as a zero-sum game between the principal and a malevolent nature. When the timing between the principal and nature is reversed, i.e. when nature decides on the worst-case process before the principal designs the contract, I show that the worst-case is always at its highest level. This analysis is carried out in Section 6.1. In Section 6.2 , I study the case when the ambiguity is about the agent's average productivity, and show that the worst-case features a productivity process which is always at it's lowest possible level. This implies that the agent is both over-compensated and over-incentivized at any point in time.

\section{Literature}

The techniques used to characterize the contract in continuous time stem from Schattler and Sung (1993) and Sannikov (2008). A related setting which features a risk neutral principal and agent and linear effort cost is studied in DeMarzo and Sannikov (2006). The existence of the over- and under-compensating regions, and the fact that the agent is over-incentivized are novel to my paper and due to the introduction of ambiguity.

Garrett and Pavan (2010a) and Garrett and Pavan (2010b) study a related setting in discrete time. In their work, the firm knows the probability law of the unknown shocks to the environment, and the manager observes the realization of the shock. Thus, the realization of the unknown process becomes private information of the agent, and Garrett and Pavan study the optimal contract which involves both moral hazard and adverse selection. In my setting, the firm has no information about the unknown process except that realizations take place in a certain interval. Also, Garrett and Pavan study only shocks to managerial productivity, while I consider both shocks to productivity and to the agent's disutility of effort. A further difference is the role of turnover in our models. While Garrett and Pavan (2010b) predict that high firing rates at the beginning of the employment relationship are optimal, my setting

suggests that under certain circumstances, firing may actually be detrimental to incentives and induce shirking.

The type of ambiguity aversion considered here is related to the maximin expected utility framework (MMEU) of Gilboa and Schmeidler (1989). In continuous time, the equivalent of Gilboa and Schmeidler's characterization has been developed by Chen and Epstein (2002), and features the requirement that all measures that are deemed possible must be equivalent to the reference measure. In the main example of my paper, the principal will be ambiguity averse with respect to agent's effort cost, and understands that nature can choose this process from a wider set than the one considered in Chen and Epstein (2002). In particular, 
different choices of nature cannot be understood as equivalent changes of measure. Therefore, the effort cost ambiguity setting cannot be subsumed under the framework of Chen and Epstein (2002). ${ }^{2}$ The case of drift ambiguity in Section 6.2 however, can be seen as a direct application, since when the principal is ambiguity averse about the drift of the output process, Girsanov's theorem implies immediately that all measures which generate the output processes the principal deems possible must be equivalent.

Recently, Epstein and Ji (2011) have studied a MMEU framework in continuous time where the ambiguity pertains to the volatility coefficient of a diffusion process, which implies that the measures are not equivalent. While I do not study this case explicitly, it is easy to see that once the technical details of the construction in Epstein and Ji (2011) are verified to hold, we should expect that the worst-case process is the one where the diffusion is at its highest possible level for all times. This is because the noisier the output process is, the more risk the principal needs to introduce into the agent's contract, which increases the probability of termination even when the agent puts in effort and thus makes the principal worse off.

The MMEU framework has been used in a series of works in contracting under ambiguity. Garrett (2009) studies a static procurement contract, and shows that ambiguity about the agent's preferences leads to the optimality of simple incentive schemes. Bergemann and Schlag (2008) and Bergemann and Schlag (2011) study a seller problem in which the principal is ambiguity averse about the buyer's valuation. They show that when the principal minimizes her regret, she can improve her payoff by offering the agent a random contract, while with ambiguity aversion alone, such improvement is not possible. In the seller problem of Bergemann and Schlag, the principal's action can be interpreted as playing a mixed strategy over the contracts offered. To compute the optimal mixed strategy, it is necessary to compute the principal's payoff for different pairs of contract and realization of ambiguity. In the dynamic framework, the space of possible contracts is too large to make this comparison feasible. Thus, while my setting incorporates a long term contract and a worst case which will change over time, it stays silent on the issue of hedging against ambiguity.

Several other papers study contracts in which the agent is ambiguity averse. Bose and Daripa (2009) show that in an auction where each buyer is ambiguity averse about the distribution of the valuation among other buyers, the seller can extract almost the total

\footnotetext{
${ }^{2}$ The framework can be forced to conform with Chen and Epstein (2002) by introducing another Brownian motion which is independent of the one in the output process and representing the agent's effort cost as a diffusion process with nature's choice as drift. Then, however, it would be necessary to ensure that both the agent's effort decision and the principal's contract cannot depend on the additional noise, which will not hold in general.
} 
surplus by offering a Dutch auction, while Di Tillio et al. (2011) show that the principal can use an ambiguous mechanism to improve her payoff. Here, an ambiguous mechanism means that the principal commits to offering the agent a set of outcome functions, depending on the agent's announcement, and it is understood that the agent is ambiguity averse about these functions. In a similar vein, Bose and Renou (2011) show that even when there is no ambiguity in the environment, the principal may choose to engineer ambiguity in the mechanism, when she knows that the agent is ambiguity averse.

Compared to these papers, my focus does not lie on exploring conditions under which the principal can improve her payoff in the presence of ambiguity aversion, but on studying how the presence of ambiguity alters the optimal contract, and how it introduces new dynamics.

\section{Model Setup}

\subsection{Technology and Utility Functions}

I study a dynamic principal agent problem. Time is continuous and infinite, and indexed by $t \geq 0$. The agent operates a project for the principal, which yields payoffs according to a diffusion process

$$
d X_{t}=\mu a_{t} d t+\sigma d B_{t}
$$

Here, $B_{t}$ is a Brownian motion defined on the probability space $(\Omega, \mathcal{F}, \mathbb{P})$ satisfying the usual conditions $^{3}$ with filtration $\mathcal{F}_{t} . a_{t} \in\{0,1\}$ is the agent's binary effort decision, which indicates whether the agent is working or shirking at time $t$. While the principal observes the output process $X_{t}$, she cannot observe the agent's effort decision. $\mu$ is the principal's average payoff given the agent exerts effort, while the term $d B_{t}$ represents the i.i.d. noise in the output process which is not due to any action of the agent. Finally, $\sigma$ measures the amount of noise present.

The principal offers the agent a contract $\alpha$, which consists of a pair of $\mathcal{F}_{t}$ adapted stochastic

processes $\left\{a_{t}, c_{t}\right\}_{t \geq 0}$ and a stopping time $\tau$. $a_{t}$ is the agent's prescribed effort process, which is either work or shirk, and $c_{t}=\int_{0}^{t} d c_{s}$ is the cumulative consumption the agent receives up to time $t$. The stopping time $\tau$ indicates when the agent is fired and the firm is terminated. In this case, both the principal and the agent receive an outside payoff which is normalized to zero. The principal has the ability to commit to any contract she offers.

\footnotetext{
${ }^{3}$ See e.g. Karatzas and Shreve (1991) for standard concepts in stochastic analysis.
} 
Both principal and agent are risk neutral. The principal receives the output from the process $X_{t}$ and makes consumption payments to the agent. Consequently, her payoff under any incentive compatible contract is

$$
J_{0}=E \int_{0}^{\tau} e^{-r t}\left(\mu a_{t} d t-d c_{t}\right)
$$

The agent on the other hand receives consumption payments from the principal, and incurs and effort cost when $a_{t}=1$. Contrary to DeMarzo and Sannikov (2006), I assume that the agent's effort cost varies over time. Let $\eta \equiv\left\{\eta_{t}\right\}_{t>0}$ denote the stochastic process governing the agent's effort cost. I assume that $\eta_{t}$ satisfies the following conditions.

Assumption 1. For all $t, \eta_{t}$ is $\mathcal{F}_{t}$ adapted and satisfies $\eta_{t} \in[\underline{\eta}, \bar{\eta}]$, with $0<\underline{\eta}<\bar{\eta}<\mu$.

The fact that $\eta_{t}$ is $\mathcal{F}_{t}$ adapted implies that the effort cost process cannot contain any additional randomness beyond what is already generated by the history of the Brownian motion $\left\{B_{t}: 0 \leq s \leq t\right\}$, which is necessary to make the principal's problem tractable. The agent's effort cost is bounded in a compact interval, with the upper bound being strictly lower than the average payoff of the project. If we had $\bar{\eta}>\mu$, the constant process $\eta_{t}=\bar{\eta}$ would imply that the principal would prefer not to execute the project even in the first best, which renders the contracting problem trivial.

The agent is more impatient than the principal and discounts time at rate $\gamma>r$. Given effort cost process $\eta$, the agent's payoff is

$$
W_{t}=E \int_{t}^{\tau} e^{-\gamma t}\left(d c_{t}-\eta_{t} a_{t} d t\right)
$$

Finally, the agent has limited liability: for all $t$, the increments in the agent's cumulative consumption payments $d c_{t}$ may not be negative.

\subsection{Effort Cost Ambiguity}

Suppose that the principal does not know the agent's effort cost. Instead, she believes that the effort cost can change over time, and follows a stochastic process $\eta \equiv\left\{\eta_{t}\right\}_{t \geq 0}$. The principal is ambiguity averse about the process $\eta_{t}$, and when choosing the contract she offers to the agent, she will maximize her payoff given the worst-case process. Alternatively, principal's ambiguity aversion can be understood as a zero-sum game between principal and nature. While the principal chooses the contract to maximize her payoffs, nature chooses 
a process $\eta$ to minimize them. Nature's choice is restricted to a process which satisfies Assumption 1 . The timing of play between the principal, nature and the agent is as follows.

Assumption 2. Let time be indexed by $T=\{-3,-2,-1\} \cup \mathbb{R}_{+}$. At $t=-3$, the principal commits to the contract $\alpha$ and offers it the agent. At $t=-2$, nature chooses $\eta$ taking $\alpha$ as given, and $\eta$ is revealed to the agent. At $t=-1$, the agent decides whether to accept or reject the contract. In case of acceptance, the contract starts and for any $t \geq 0$ the agent makes his effort decision taking the processes $\alpha$ and $\eta$ as given. In case of rejection both principal and agent receive their outside payoff of zero.

Since the agent is ambiguity neutral, he will have to learn the process $\eta$ in order to be able to compute his expected utility. Thus, the assumption that he learns $\eta$ before the contract starts is necessary for the problem to be well defined. Whether $\eta$ is revealed to the principal however is inconsequential, since given any contract $\alpha$, the principal can simply compute nature's best response. Thus, she will have knowledge of nature's choice in equilibrium.

When studying the principal's problem, I rule out mixed strategies for both the nature and the principal. The reason for this is tractability. As Bergemann and Schlag (2008) have shown, the principal could improve her payoff in the presence of ambiguity by randomizing over the contract she offers to the agent. In my setting, to compute the principal's payoff from such a strategy, it is necessary to compute the principal's payoff given an arbitrary combination of contract $\alpha$ and effort process $\eta$, and to compare payoffs across pairs of $(\alpha, \eta)$. For arbitrary stochastic processes $\alpha$ and $\eta$, this problem is too complex to yield a concrete solution.

Note that the assumption does not imply that the contract itself is not random. Since $\alpha$ and $\eta$ are $\mathcal{F}_{t}$ adapted, the contract will still depend on the noise of the output process. However, neither principal nor nature can introduce any additional uncertainty.

Under ambiguity aversion, the principal's problem is to maximize her utility under the worstcase process, subject to the law of motion of the output, and the incentive compatibility and participation conditions of the agent.

$$
\begin{aligned}
& J_{0}=\sup _{\alpha} \inf _{\eta} E \int_{0}^{\tau} e^{-r t}\left(\mu a_{t} d t-d c_{t}\right) \\
& \text { s.t. } \\
& d X_{t}=\mu a_{t} d t+\sigma d B_{t}
\end{aligned}
$$


Given the contract $\alpha$ and effort cost process $\eta$, the agent's continuation utility at time $t$ satisfies

$$
W_{t}=E \int_{t}^{\tau} e^{-\gamma(s-t)}\left(d c_{s}-\eta_{s} a_{s} d s\right)
$$

The timing structure above corresponds most closely to the way ambiguity aversion is represented in the decision theoretic literature, see Gilboa and Schmeidler (1989). When the timing of principal and nature is reversed, the principal can potentially be better off. Since nature chooses first, the principal can take nature's choice as given and condition the contract on the process $\eta$. In Section 6.1 I show that this indeed yields to an improvement for the principal.

\section{Optimal Contract}

In this section, I derive the optimal contract under the timing assumption 2. The worst-case process is not constant, and will vary depending on the past performance of the agent. There are two regimes in the contract. An agent with a sufficiently high past performance will find himself in the over-compensating regime. In this case, ambiguity forces the growth of his continuation value to be relatively high, and in expectation the agent receives high payouts. When the agent's past performance is sufficiently low, he will enter the under-compensating regime, in which the growth of his continuation value and therefore his expected payouts are relatively low.

Both regimes exhibit persistence - in expectation, the longer an agent has been in the overcompensating regime, the less likely it is that he will ever leave it. Similarly, once agents enter the under-compensating regime, they expect to stay there. In both cases, however, the agent receives excessively strong incentives. This is because the principal fears that unless the agent's risk exposure is at the highest possible level, nature can find a way to induce the agent to shirk.

\subsection{Incentive Compatibility}

I begin my analysis of the contract with the agent's continuation value, and an a-priori result on the optimal contract. Suppose that the principal designs a contract which induces a type $\tilde{\eta}$ to not participate. Since the principal's payoff is zero if the agent rejects the contract, we

know that at the optimal contract $J_{0} \geq 0$. But then nature can just choose $\tilde{\eta}$ and guarantee a zero payoff to the principal. In order to prevent this, the principal's optimal contract can 
never exclude any type.

Lemma 3. At any optimal contract

$$
W_{0}=E\left(\int_{0}^{\tau} e^{-\gamma t}\left(d c_{t}-\eta_{t} a_{t} d t\right)\right) \geq 0
$$

for all $\eta \in H$.

In DeMarzo and Sannikov (2006) as well as numerous other works in dynamic contracting, the agent's continuation value plays a crucial role in determining the properties of the contract. In the case without ambiguity, it can be shown that the continuation value

$$
W_{t}=E\left(\int_{t}^{\tau} e^{-\gamma(s-t)}\left(d c_{s}-\eta_{s} a_{s} d t\right)\right)
$$

summarizes the entire history of the contract, and can thus be taken as a state variable in the principal's optimization problem.

When the principal is ambiguity averse, this approach is still valid. For every contract, there exists a worst-case effort cost process $\eta$ and the principal's payoff can be calculated as-if she assumes that the agent's true effort cost is actually $\eta$. Then, the agent's continuation value can be decomposed according to the martingale representation theorem taking $\eta$ as given.

Proposition 4. At any optimal contract the agent's continuation value evolves according to

$$
d W_{t}=\left(\gamma W_{t}+\eta_{t} a_{t}\right) d t+\psi_{t} d B_{t}
$$

Unlike in the case without ambiguity, $W_{t}$ is not the actual continuation value of the agent, since the principal and the agent's knowledge about $\eta$ will differ. Instead, $W_{t}$ describes the agent's continuation value as-if the principal's worst-case belief $\eta$ were true, and is used by the principal solely to characterize the form of the contract.

\subsection{Optimal Contract when the Principal Moves First}

Under Assumption 2, the principal chooses an effort process $a_{t}$ and a consumption process $c_{t}$ for the agent, such that given the worst-case realization of $\eta$, the agent will put in effort. ${ }^{4}$

\footnotetext{
${ }^{4}$ From Zhu (2011), we know that the contract which induces the agent to always exert effort may not be optimal, and instead, the principal can increase her payoff by allowing the agent to shirk when he is close to termination. The main results in my paper would continue to hold if I allowed for shirking along the optimal contract, and I omit the case with shirking for the sake of simplicity and expositional clarity.
} 
From the martingale representation in Proposition 4, this is equivalent to setting a consumption policy $c_{t}$ and a risk exposure process $\psi_{t}$. Unlike in the standard contracting problem, the principal's choice of the contract is not driven by incentive considerations alone, and she will have to take into account how ambiguity may compromise the agent's decision.

We can heuristically derive a solution to the principal's problem as follows. First, the agent shirks whenever $\psi_{t}<\frac{\sigma}{\mu} \eta_{t}$. When shirking in a small time interval $(t, t+d t)$, he saves $\eta_{t} d t$ in effort cost. However, the expected change in the output process is lower by $\mu d t$, which is equivalent to the Brownian noise having a realization which is lower by $\frac{\mu}{\sigma} d t$ on average. This implies that his expected continuation value $W_{t}$ falls by $\frac{\mu}{\sigma} \psi_{t}$. The agent will only shirk when this tradeoff is positive, or $\eta_{t}-\frac{\mu}{\sigma} \psi_{t}>0$.

Now, suppose that for a small interval of time $d t$, the principal sets $\psi_{t}$ at some value such that $\psi_{t}<\frac{\sigma}{\mu} \bar{\eta}$ on $(t, t+d t)$. If the worst-case ambiguity process is such that

$$
\eta_{t}>\psi_{t} \frac{\mu}{\sigma}
$$

the agent will not work, and the principal loses $\mu d t$ in output. At the same time, however, since the principal can anticipate the agent's response given $\eta_{t}$, she knows that the agent does not work on $(t, t+d t)$. Thus, she does not need to compensate the agent for his effort, and the growth of $W_{t}$ between $t$ and $t+d t$ falls by $\eta_{t} d t$ in expectation. Since $W_{t}$ is a state variable for the principal, she values the savings in compensation by approximately $J^{\prime}\left(W_{t}\right)$. Thus, if the principal sets $\psi_{t}<\frac{\sigma}{\mu} \bar{\eta}$, nature can either induce the agent to shirk, in which case the principal's utility changes by $-\left(\mu+J^{\prime}(W) \eta_{t}\right) d t$ or nature can choose an $\eta_{t} \leq \psi_{t} \frac{\mu}{\sigma}$, so that the agent works. In general, $J^{\prime}(W)$ can be either positive or negative. Let us conjecture that the principal's value function has the same shape as in DeMarzo and Sannikov (2006), that is, it is concave, first increasing and then decreasing, and satisfies $J^{\prime}(\bar{W})=-1$, where $\bar{W}$ is the maximal continuation value of the agent in the contract. We have $\mu+J^{\prime}(W) \eta_{t} \geq \mu-\bar{\eta}$ since $J^{\prime}(W) \geq-1$ on $(0, \bar{W})$ by the concavity of the principal's value function. Because we assumed that the project is profitable in the first best, we have $\mu>\bar{\eta}$, and this implies that the principal will always prefer to reap the project's benefits and compensate the agent for effort rather than have the agent put in no effort. Since nature wants to minimize the principal's payoff, she will therefore choose an $\eta$ such that the agent shirks whenever $\psi_{t}<\frac{\sigma}{\mu} \bar{\eta}$. The principal, acting as a Stackelberg leader, foresees this and sets the agent's risk exposure at the maximal level for all $t$, which is the only way to prevent the agent from shirking under the worst case. Thus, we know that $\psi_{t}=\frac{\sigma}{\mu} \bar{\eta}$ in the optimal contract.

Given that the principal prevented nature from choosing an ambiguity process such that 
the agent shirks, what will the choice of $\eta_{t}$ be? Although nature cannot affect $a_{t}$, she can still lower the principal's utility via the amount the principal has to pay to compensate the agent. Thus, when $J^{\prime}\left(W_{t}\right)<0$, and the principal loses utility the more she has to compensate the agent for effort, nature will set $\eta_{t}=\bar{\eta}$ to maximize the losses. On the other hand, if $J^{\prime}\left(W_{t}\right)>0$, which happens when the agent's continuation value is low, the probability of project termination is very high. In this case, the principal actually benefits from $W_{t}$ having a higher expected growth rate, because the reduction in the likelihood of inefficient project failure will outweigh the expected cost of compensating the agent for effort. Nature will choose $\eta_{t}$ accordingly, and set it at the lowest possible value, so that $\eta_{t}=\underline{\eta}$ whenever $J^{\prime}\left(W_{t}\right)<0$.

In sum, the principal will always choose the strongest possible incentives for the agent to insure against ambiguity, and the worst-case type will be the one with the lowest effort cost whenever $J^{\prime}(W)>0$ and the highest effort cost when the opposite is true.

To show the result rigorously, it is necessary to establish both that the principal's value function solves a variant of the HJB equation which accounts for the ambiguity, and that $J$ indeed has the conjectured shape. This is carried out in the following Proposition.

Proposition 5. When ambiguity is revealed after the principal chooses the contract, the principal's value function satisfies the HJB equation

$$
r J\left(W_{t}\right)=\sup _{\psi_{t}} \inf _{\eta_{t}} \mu a\left(\psi_{t}, \eta_{t}\right)+J^{\prime}\left(W_{t}\right)\left(\gamma W_{t}+\eta_{t} a\left(\psi_{t}, \eta_{t}\right)\right)+\frac{1}{2} J^{\prime \prime}\left(W_{t}\right) \psi_{t}^{2}
$$

almost every where in $W$ with the boundary conditions $J(0)=0$ and $J^{\prime}(\bar{W})=-1$. a $\left(\psi_{t}, \eta_{t}\right)$ is given by

$$
a\left(\psi_{t}, \eta_{t}\right)=1\left\{\psi_{t} \geq \frac{\sigma}{\mu} \eta_{t}\right\}
$$

and we have

$$
\psi_{t}=\bar{\psi} \equiv \frac{\sigma}{\mu} \bar{\eta}
$$

for all $t$ and

$$
\eta_{t}= \begin{cases}\bar{\eta} & \text { if } W \geq W_{0} \\ \underline{\eta} & \text { if } W<W_{0}\end{cases}
$$

where $W_{0}$ is the continuation value for which $J^{\prime}\left(W_{0}\right)=0$. Further, $J$ is concave for all $W$, and $d c_{t}=0$ for all $W_{t}<\bar{W}$. 
The most important feature of the contract under ambiguity is that the agent's employment contract is characterized by two different regimes. For the sake of interpretation, assume that the principal believes that $\eta_{t}$ is the worst-case process while the agent's actual effort cost process is $h_{t} \in(\underline{\eta}, \bar{\eta})$.

When $W>W_{0}$, the principal will believe that $\eta_{t}=\bar{\eta}$ and the agent is very difficult to incentivize. The agent's compensation scheme will feature a high drift in $W_{t}$, which can be seen from setting $\eta_{t}=\bar{\eta}$ in equation (7). The contract features a high expected payoff for the agent, in order to compensate him for effort. Also, since $\psi_{t}$ is at the maximal level, the agent will be faced with powerful incentives, and the compensation contract will feature a high pay-performance sensitivity.

Because $h_{t}<\bar{\eta}$, the principal will both over-compensate and over-incentivize the agent, that is, she will promise the agent higher expected payoffs than would be necessary to compensate for his effort, and the agent's incentives are stronger than then would have to be if the contract were geared to the actual effort cost $h_{t}$. Since the drift of $W_{t}$ is positive and relatively high due to the term $\bar{\eta}$, the probability of falling below $W_{0}$ and into the other regime is relatively low. Hence, once the agent reaches a high continuation value, he is also likely to stay there.

On the other hand, when $W_{t}$ is below $W_{0}$, the principal will believe that the agent is relatively easy to compensate, but will still shirk if the risk exposure is anything but the highest level. Thus, although the agent receives very powerful incentives, he will reap low benefits, and will be under-compensated compared to the case without ambiguity. In expectation, his payouts are small, and his firing probability is relatively large. Because of this, he is less likely to move up to the regime in which he is over-compensated.

Figure 1 illustrates this result.

The existence of two regimes in the agent's compensation scheme holds for all specifications of ambiguity in which $\bar{\eta}>\underline{\eta}$. When ambiguity increases, that is when the distance between $\bar{\eta}$ and $\underline{\eta}$ becomes larger, so does the persistence of the two regimes. The agent who is stuck in the under-compensating region will receive an even lower drift in continuation value, and has thus a lower chance of reaching the over-compensating regime. When the agent is in the latter, his growth in $W$ will be even higher, which implies more payouts and a lower likelihood to fall back to the under-compensating regime. For both cases, however the strength of incentives increases. 


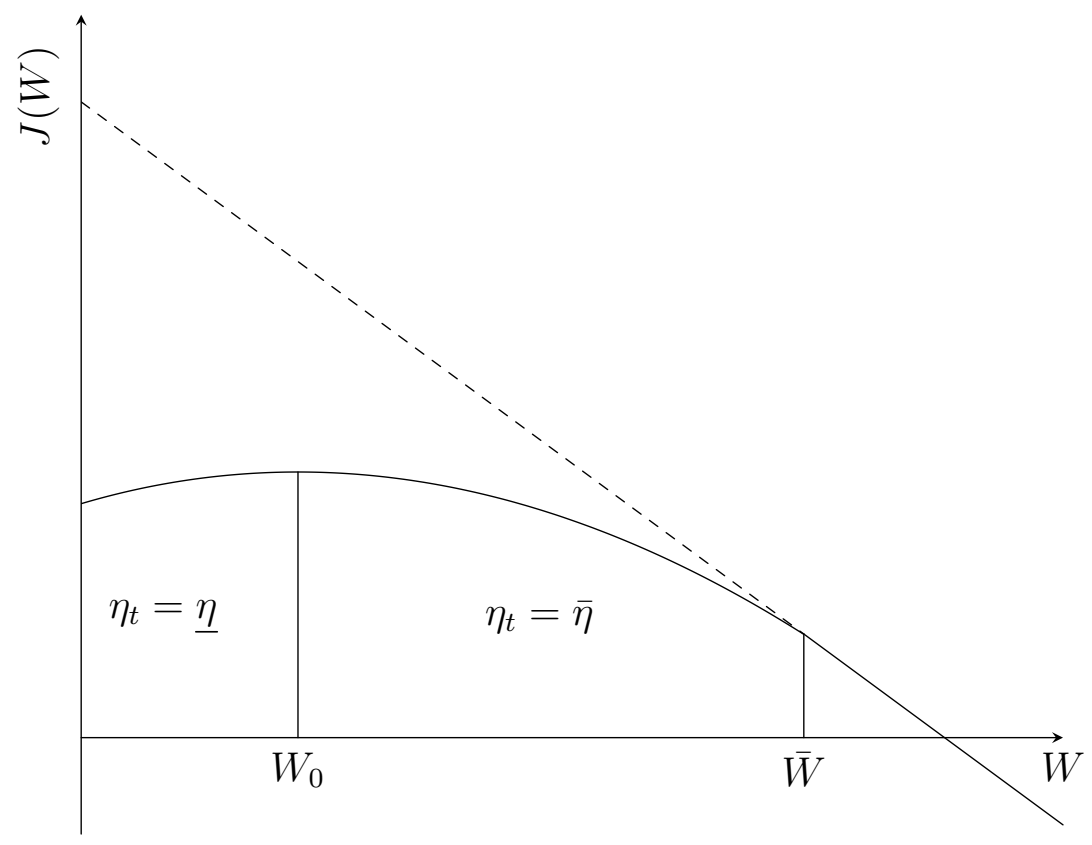

Figure 1: Shape of $J(W)$ and $\eta(W)$

\subsection{Implementation}

From the characterization of the optimal contract, the we know that the growth of the agent's continuation value is lower in the under-compensating regime, since the principal believes that $\eta_{t}=\underline{\eta}$. Generally, it is possible to interpret the agent's continuation value in a dynamic contracting environment as the amount of cash the firm has at hand, either in a money market account as in DeMarzo et al. (2010), or as a draw on a credit line as in DeMarzo and Sannikov (2006). Consequently, we can identify the expected growth of the firm's cash stock as $\gamma W+\eta(W)$, and at the optimal contract, the growth of the firm's free cash will be lower when the cash stock itself is low, or alternatively, when the draw on the firm's credit line is close to the limit.

This implies that a firm with low cash holdings and relatively high bankruptcy probability will optimally choose to have lower growth in its cash stock. Fixing $W_{t}$, the difference in growth rates in the different regimes is $(\bar{\eta}-\eta) d t$. Thus, when $W_{t}$ is low, the firm burns cash at rate $(\bar{\eta}-\underline{\eta}) d t$. Although this practice seems bad for the firm, since it increases the likelihood of bankruptcy, it is reminiscent of various behaviors observed in practice. Stock buybacks for instance often occur when the stock price is low, which in our model corresponds to a low value for the principal, and take additional money out of the firm's coffers. Similarly, while the firm would buy back stocks when $W_{t}$ is low, the literature documents that firms 
after sufficiently high performance are more likely to issue dividends, which occurs exactly when $W_{t}$ hits $\bar{W}$.

For this interpretation to make sense, however, one needs to be very careful for how a buyback affects managerial incentives. After all, whether the shares are retired or not, it affects either the fraction of outstanding shares the manager is holding, or the allocation of payouts to the manager whenever dividends are paid. Thus, in general, the firm would need to lower the manager's share holdings when a buyback occurs in order to not compromise incentives.

A simpler solution which does not require the adjustment of the manager's equity share is for the firm to enter a performance sensitive debt contract on its long term debt. In this scenario, the firm has a credit line to manage day-to-day expenses, and the manager's continuation value can be explained as a function of the draw on the credit line. At the same time, the firm has long term debt in place which exhibits a varying repayment rate. When $W_{t}$ is low, which happens after poor performance of the firm, the interest on the debt is high, while when $W_{t}$ is high, the interest rate is relatively low. This way, performance sensitive debt can be used to fine-tune the firm's cash flows in order to incentivize the agent under the worst case realization of ambiguity. The proposition below shows that we can indeed implement the optimal contract with a long term debt whose interest rate exhibits performance dependence.

Proposition 6. Suppose the firm has long term debt D, a credit line $C_{L}$ with constant interest $\gamma$ and equity. Suppose that the equity share of the agent is $\Psi=\psi \frac{1}{\sigma}$, the amount of long term debt equals

$$
D=\frac{1}{r}\left(\mu-\frac{h}{\Psi}-\gamma C_{L}\right)
$$

for some $h \in(\underline{\eta}, \bar{\eta})$ and the long term debt is performance sensitive. Whenever $W_{t}>W_{0}$ the interest on the debt is $r_{1}=\frac{\mu-\gamma C_{L}-\frac{\bar{\eta}}{\Psi}}{D}<r$ while when $W_{t} \leq W_{0}$ the interest is $r_{2}=$ $\frac{\mu-\gamma C_{L}-\frac{\eta}{\gamma}}{D}>r$. Then the optimal contract is implemented and

$$
W_{t}=\Psi\left(C_{L}-M_{t}\right)
$$

where $C_{L}=\frac{\bar{W}}{\Psi}$.

Proof. Let $p_{t}$ be the instantaneous debt repayment rate. We have

$$
d M_{t}=\gamma M_{t} d t+p_{t} d t+d D i v_{t}-d X_{t}
$$


The debt repayment equals $r_{t} D$ so that

$$
d M_{t}=\gamma M_{t} d t+\left(\mu-\frac{1}{\Psi} \eta\left(W_{t}\right)-\gamma C_{L}\right) d t+d D i v_{t}-d X_{t}
$$

Using guess and verify, the agent's HJB equation must solve

$$
\gamma W_{t}=\max _{a \in\{0,1\}}-\Psi\left(\gamma M_{t}+\mu-\frac{1}{\Psi} \eta\left(W_{t}\right)-\gamma C_{L}+\frac{d D i v_{t}}{d t}-\mu a\right)+\frac{d c_{t}}{d t}-\eta\left(W_{t}\right) a
$$

and setting $d c_{t}=\Psi d D i v_{t}$ implies the result.

A crucial feature of this implementation is that performance sensitive debt is not chosen to incentivize the agent to exert effort, as is the case in Piskorski and Tchistyi (2011), since the managerial equity share already serves this function. Instead, performance sensitive debt is used to adjust the growth of the manager's continuation value in order to prevent the manager from reaping excessive benefits under the worst-case prior.

\section{Agent's Value Function}

In the case where the principal does not learn the agent's type $\eta$, the principal will implement the contract designed for the worst case type. It is irrelevant whether the worst case process is the actual effort cost process of the agent as long as the principal believes that it is. In this section, I explicitly construct the agent's optimization problem when the principal is wrong about the agent's true effort cost, under the assumption that the latter takes a constant value. ${ }^{5}$ As Oyer and Schaefer (2011) point out, measuring characteristics such as effort cost or risk aversion for a particular employee who is given a contract is not feasible for empirical researchers. We should expect that the firms who hire employees also have only imprecise knowledge about these characteristics, and thus will offer contracts which are not perfectly matched to the agent. Hence, the question of how an agent behaves under a contract which is intended for the worst-case type is of practical importance.

Under the worst-case contract, even though the agent will receive excessive pay-performance sensitivity, when the effort cost is sufficiently close to $\bar{\eta}$, the agent will still choose to shirk when $W_{t}$ is low and he is close to the firing boundary. This is because firing can actually decrease the agent's incentives to exert effort. An agent with a low $W_{t}$ expects to be fired

\footnotetext{
${ }^{5}$ This assumption is for tractability, since it is difficult to characterize a solution to the agent's problem for more general processes.
} 
with a high probability before he reaches the payment boundary $\bar{W}$ even when he puts in effort. This, together with the fact that he needs to go trough the under-compensation regime before reaping any rewards will decrease the marginal value of effort. When the effort cost is sufficiently high, these two factors are sufficient to discourage the agent from exerting effort.

This result further exacerbates the persistence of the under-compensation region. Since the agent shirks, he is further lowering the drift of the continuation value and if $W_{t}$ is sufficiently low the drift will be negative. Thus, the agent is shirking while expecting to get fired in the future, and the only thing to prevent firing is a sufficiently high shock to the output process. To solve the agent's problem, it is first necessary to characterize how the contract reacts when the agent shirks, so that we can compute the agent's tradeoff between working and shirking at different times. Since the environment is common knowledge, the agent understands that the principal will observe the cash flows, and remunerate the agent as-if he had type $\eta_{t}=\eta\left(W_{t}\right)$, where $\eta\left(W_{t}\right)$ is as in Proposition 5. In the principal's contract, the agent's continuation value under the worst case type is used as a state variable and hence summarizes the dynamics of the contract for each possible history of output. Therefore, the agent can use $W_{t}$, the principal's belief about the agent's continuation value, to track how the contract changes over time.

Thus, even though the agent's true continuation value, which is given by

$$
V_{t}=E \int_{0}^{\tau} e^{-\gamma t}\left(d c_{t}-h a_{t} d t\right)
$$

is different from the principal's estimate $W_{t}$, the agent will still use it to compute how the contractual parameters change with effort, and the law of motion for $W_{t}$,

$$
d W_{t}=\left(\gamma W_{t}+\eta\left(W_{t}\right) a_{t}\right) d t+\bar{\psi} a_{t}^{P} d B_{t}
$$

governs the dynamics of the agent's problem. Here, I have used $a_{t}^{P}=1$ to denote the effort demanded by the principal at time $t$, which may be different from the agent's actual effort, $a_{t}$.

When the agent shirks, the principal and the agent's information sets, $\mathcal{F}_{t}^{P}$ and $\mathcal{F}_{t}^{A}$ will differ not only in the assessment of $\eta_{t}$ but also in the drift of the process $d X_{t}$. If the agent shirks, he will know that $d X_{t}$ follows

$$
d X_{t}=\sigma d B_{t}
$$


The principal on the other hand cannot observe the output process, and hence still assumes that the agent is putting in effort. Given her information set, $d X_{t}$ follows

$$
d X_{t}=\mu d t+\sigma d B_{t}
$$

Both principal and agent's filtrations are associated a Brownian Motion $B_{t}^{P}$ and $B_{t}^{A}$. Using Girsanov's Theorem, we can relate these two by

$$
d B_{t}^{A}=d B_{t}^{P}-\frac{\mu}{\sigma}\left(a_{t}-a_{t}^{P}\right) d t
$$

The intuition for the difference between $B_{t}^{P}$ and $B_{t}^{A}$ is as follows. When the agent shirks, between $t$ and $t+d t$ the output will be lower by $\mu d t$. Suppose the change in output is $X_{t+d t}-X_{t}$. The agent knows that the actual realization of the Brownian motion is

$$
B_{t+d t}-B_{t}=\frac{1}{\sigma} X_{t+d t}-X_{t}
$$

The principal, not knowing that the agent shirked, will conclude that the realization of the noise process has been

$$
B_{t+d t}-B_{t}=\frac{1}{\sigma}\left(X_{t+d t}-X_{t}\right)-\frac{\mu}{\sigma} d t
$$

Thus, the principal will attribute the low output to a particularly low realization of $B_{t+d t}-B_{t}$, and will adjust the contract accordingly.

Thus, under the agent's information set and given possible shirking $W_{t}$ follows

$$
\begin{aligned}
d W_{t} & =\left(\gamma W_{t}+\eta\left(W_{t}\right)\right) d t+\frac{\sigma}{\mu} \bar{\eta} d B_{t}^{A} \\
& =\left(\gamma W_{t}+\left(\eta\left(W_{t}\right)-\bar{\eta}\right)+\bar{\eta} a_{t}\right) d t+\bar{\psi} d B_{t}^{P}
\end{aligned}
$$

Hence, if the agent shirks when the principal would have mandated working, the drift of the continuation value $W_{t}$ diminishes by $\bar{\eta}$.

Having defined the effect of shirking on the continuation value, we can now formulate the agent's HJB equation, which determines the solution to the agent's decision.

Proposition 7. Suppose that the agent's effort cost is a constant $h$. The agent's value function $V_{t}=V\left(W_{t}\right)$ is $C^{1}$ and satisfies almost everywhere the HJB equation

$$
\gamma V\left(W_{t}\right)=\max _{a_{t}}-h a_{t}+V^{\prime}\left(W_{t}\right)\left(\gamma W_{t}+\left(\eta\left(W_{t}\right)-\bar{\eta}\right)+\bar{\eta} a_{t}\right)+V^{\prime \prime}\left(W_{t}\right) \frac{1}{2} \bar{\psi}^{2}
$$


with the boundary conditions $V(0)=0$ and $V^{\prime}(\bar{W})=1$. The agent exerts effort whenever

$$
V^{\prime}(W) \geq \frac{h}{\bar{\eta}}
$$

There is a $\hat{h}$ such that for all $h \leq \hat{h}$, the agent always exerts effort, and for $h>\hat{h}$, the agent shirks when $W \in\left[0, W_{s}(h)\right]$ for some $W_{s}(h)<W_{0}$ and works for all $W>W_{s}(h)$. Further, for $h>\tilde{h}>\hat{h}, V$ is globally concave, while for any $h \leq \tilde{h} V$ is convex on $\left[0, W_{0}\right]$. For any $h, V$ is concave on $\left[W_{0}, \bar{W}\right]$.

In the above formulation, the strength of the agent's incentives at time $t$ is given by $V^{\prime}\left(W_{t}\right)$, and the agent will only put in effort at $t$ when $V^{\prime}\left(W_{t}\right)$ is sufficiently high. In the case without ambiguity, where $\eta_{t}=h$, the agent's value function satisfies $V_{t}=W_{t}$ and therefore $V^{\prime}\left(W_{t}\right)=1$, so that the agent will always exert effort.

We may have guessed that the agent always puts in effort since the agent's risk exposure $\frac{\sigma}{\mu} \bar{\eta}$ is greater than $\frac{\sigma}{\mu} h$, i.e. the agent is over-incentivized. This turns out to be wrong for agents which have a sufficiently high effort cost. The effort decision is driven by to features of the contract. When $W$ reaches its upper boundary, the agent gets paid a discrete amount, while when $W$ reaches zero, he gets terminated, and never receives payoffs again. Exerting effort implies a higher drift in $W$, so that in expectation, an agent exerting effort receives more payments, and has a lower firing probability.

Potential termination of the agent however is a double edged sword when it comes to incentives. If $W$ is close to zero and the firing probability high, an agent who puts in effort may still have a relatively high probability of being terminated. For such an agent, reaching the payment boundary $\bar{W}$ is relatively unlikely. Thus, the marginal payoff to effort will be relatively low, and if $h$ is sufficiently high, the agent will not find it optimal to exert effort, even though his pay performance sensitivity is high.

In this case, the agent will shirk, and from inspecting the HJB equation, we can see that the drift of his continuation value process will be negative. In expectation, the agent knows that since he is not working, he will eventually face termination. The only way for him to ever receive payoffs is a sequence of sufficiently high shocks to the output process - in other words, luck. Consequently, the agent's value function will be convex on this region, since he will benefit from a more risky output process. ${ }^{6}$ Since the possibility of termination is

\footnotetext{
${ }^{6}$ The reason why an agent who is not getting paid and knows that in expectation he will be fired before he gets any payments is not leaving the firm immediately is precisely because it is optimal for him to gamble on a sufficiently high sequence of outputs which will take him into a region where its optimal to exert effort again.
} 
detrimental to such an agent's effort decision, $V^{\prime}(W)$ will be higher the further away the agent is from termination, until some value $W_{s}$ is reached at which the agent starts working. When $h$ is low, the firing probability is not sufficient to discourage the agent from working, even when $W$ is close to zero. The drift of $W_{t}$ will always be positive, and the agent knows that in expectation, he will reach both the over-compensation regime and ultimately the payment boundary. Such an agent does not benefit from additional risk exposure, since the downside risk of a higher firing probability outweighs the potential benefit of getting faster to the payment boundary, and the value function will be globally concave. While an agent with high $h$ benefits from excessive risk exposure at least for small $W$, for a low effort cost agent, the additional risk exposure $\bar{\psi}$ is detrimental.

When $W$ reaches $W_{0}$, the agent is both-over incentivized and over-compensated relative to his effort cost, and he will exert effort. Exerting effort increases the likelihood of reaching this region and decreases the expected duration until it is reached. For some values of $W$ left of $W_{0}$, this incentive will be sufficient to motivate the agent to work, and we have $W_{s}<W_{0}$ for all $h$.

In line with the previous intuition, once the agent is in the over-compensating scheme, he should be risk averse. After all, he is already putting in effort and will expect to remain in the favorable regime if risk is sufficiently low. Thus, once $W>W_{0}$, the downside risk of being demoted to the under-compensating region due to a sequence of negative shocks outweighs the potential benefit of reaching the payment boundary faster.

But risk aversion implies that $V^{\prime}(W)$ will be decreasing in $W$. Thus, when $W$ is close to $\bar{W}$ a higher continuation value will imply weaker incentives. In this situation, the probability of being terminated before being compensated is relatively low, and even if the agent does not put in effort, the expected growth of his continuation value will be large, because of the term $\gamma W_{t}$ in the drift of $d W_{t}$ in equation 7 . Thus, the agent expects to get paid whether he puts in effort or not. Unlike in the case where the agent is close to firing, this decrease in the effectiveness of the agent's incentives will not be sufficient to cause the agent to shirk, and the agent will always put in effort when $W_{t}$ is sufficiently high. Unless the effort cost of the agent is very low, and the value function is globally concave, the agent's incentives will be strongest when the continuation value is in the middle, either right before or right after he has reached the over-compensating region.

Figure 2 displays the agent's value function as well as its slope, which indicates when the agent puts in effort, for high, medium and low effort cost values. In the upper panels, the dashed line is the identity, at which $V(W)=W$. Thus, depending on the effort cost as well 

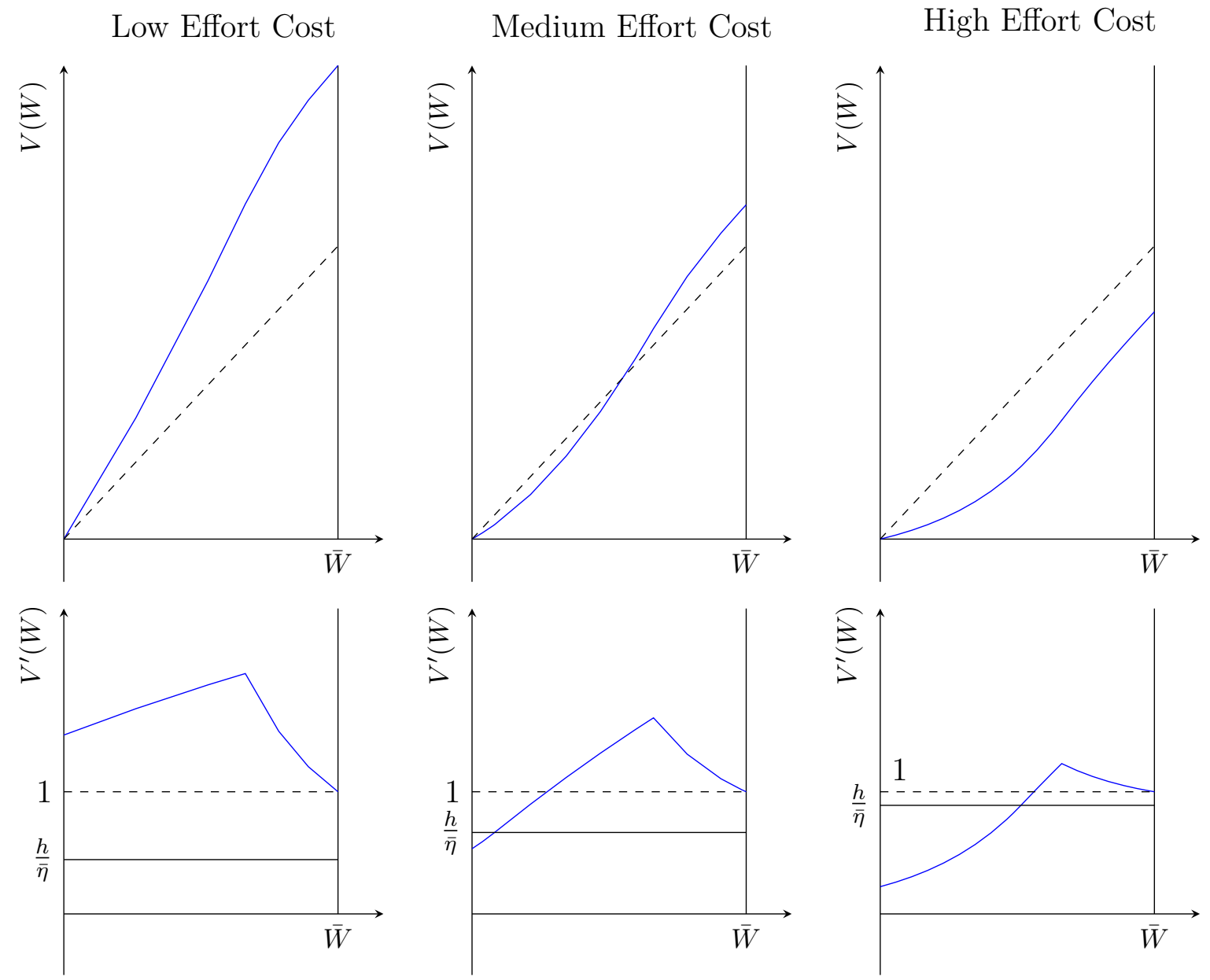

Figure 2: Agent's Value Function and Incentives 
as the current $W$, the agent's value function may be higher or lower than the continuation value the principal believes the agent to have.

In the lower panels the solid black line corresponds to $\frac{h}{\bar{\eta}}$, and the agent works whenever $V^{\prime}(W)$ is above the line. Consistent with the boundary condition $V^{\prime}(\bar{W})=1$, the slope of the agent's value function converges to one as $W$ approaches $\bar{W}$ for any effort cost.

\section{Extensions}

\subsection{Contract when Nature Moves First}

In this section, I study the contract when the timing between the principal and nature is reversed, and the timing assumption now reads

Assumption 8. Let time be indexed by $T=\{-3,-2,-1\} \cup \mathbb{R}_{+}$. At $t=-3$, nature chooses $\eta$. At $t=-2$, the principal observes $\eta$ and commits to contract $\alpha$ and offers it to the agent. At $t=-1, \eta$ is revealed to the agent and the agent decides whether to accept or reject the contract. In case of acceptance, the contract starts and for any $t \geq 0$ the agent makes his effort decision taking the processes $\alpha$ and $\eta$ as given. In case of rejection both principal and agent receive their outside payoff of zero.

When the principal knows the realization of the worst-case process before she makes her own choice, she has an advantage compared to the case when nature makes her choice after the principal chooses the contract. Instead of nature adapting the worst case to the principal's contract, the principal can condition the contract on the choice of nature. Thus, nature's ability to manipulate the principal's payoff is diminished.

Just as in Section 4.2, the principal ensures that the agent exerts effort in equilibrium. Similarly, the principal is risk averse because the contract involves inefficient termination when the agent's continuation value reaches zero. Nature's choice of of $\eta$ affects the principal's payoff both through the cost of compensating the agent for effort, as well as the cost of making the contract react more strongly to the noise in the output process.

When $J^{\prime}(W)<0$, nature will want to set $\eta_{t}=\bar{\eta}$ since both the agent's risk exposure and expected compensation are increasing in $\eta_{t}$, and are both detrimental for the principal. When $J^{\prime}(W)>0$, the tradeoff will be more complicated. The principal will benefit from a higher effort cost, since it moves the agent's continuation value away from the termination boundary. However, because the principal is risk-averse in equilibrium, nature knows that 
for any choice of $\eta_{t}$, the principal will set $\psi_{t}$ at the lowest possible level which still guarantees

effort. Hence, the principal's best response is $\psi_{t}=\frac{\sigma}{\mu} \eta_{t}$, and if nature sets a small $\eta_{t}$, the risk in the contract will diminish, which will benefit the principal.

Thus, nature will have to trade off a lower effort cost versus a higher risk exposure for the principal when $J^{\prime}$ is negative. In the proposition below, I show that this tradeoff will always be in favor of a high effort cost. That is, for any $W$ such that $J^{\prime}(W)<0$, the principal's risk aversion outweighs possible benefits of having a higher drift in $W_{t}$ and nature will set $\eta_{t}=\bar{\eta}$.

Proposition 9. Suppose that the principal knows the ambiguity process $\left\{\eta_{t}\right\}$ before choosing the contract. Then, the principal's value function solves the HJB equation

$$
r J(W)=\min _{\eta} \max _{\psi} \mu a(\psi, \eta)+J^{\prime}(W)(\gamma W+\eta a(\psi, \eta))+J^{\prime \prime}(W) \frac{1}{2} \psi^{2}
$$

subject to the boundary conditions $J(0)=0$ and $J^{\prime}(\bar{W})=-1$, where

$$
a(\psi, \eta)=1\left\{\psi \geq \frac{\sigma}{\mu} \eta\right\}
$$

At the optimal contract the risk exposure is given by

$$
\psi=\bar{\psi} \equiv \frac{\sigma}{\mu} \bar{\eta}
$$

and the worst-case process follows

$$
\eta_{t}=\bar{\eta}
$$

The value function is concave everywhere and $J^{\prime}(W)>0$ for all $W<W_{0}$ for some $W_{0} \in$ $(0, \bar{W})$, while $J^{\prime}(W)<0$ for all $W>W_{0}$.

Unlike in the case in Section 4.2, the agent always receives the maximal compensation, and the effort cost is always at the highest level. Thus, the result on the under- and overcompensating regions in Proposition 5 does not carry over.

In Section 5, I have studied the incentives of an average agent with constant effort cost $h \in(\underline{\eta}, \bar{\eta})$ which is subjected to the principal's worst-case contract. The main result is that the agent's incentives when $W$ is low may not be sufficient to ensure the agent puts in effort, while they always be sufficiently high when $W_{t}$ is high. When ambiguity is revealed before the contract is chosen by the principal, the under-compensating region disappears and agent 
is left with a contract that both over-compensates and over-incentivizes him. It is thus not surprising that the worst-case incentives are strong enough to ensure that any agent with constant effort cost $h$ will put in effort forever, and he will reap excessive payoffs for doing so.

Proposition 10. Suppose that ambiguity is revealed before the contract. The agent's value function solves

$$
\gamma V(W)=\max _{a \in\{0,1\}}-h a+V^{\prime}(W)(\gamma W+\bar{\eta} a)+V^{\prime \prime}(W) \frac{1}{2} \bar{\psi}^{2}
$$

subject to the boundary conditions $V(0)=0$ and $V^{\prime}(\bar{W})=1$, where $\bar{\psi}=\frac{\sigma}{\mu} \bar{\eta}$. The agent puts in effort forever and the agent's value function admits the closed form solution

$$
V=C\left(W+\frac{\bar{\eta}}{\gamma}\right) \int_{0}^{W} \frac{\exp \left(-\frac{1}{\frac{1}{2} \bar{\psi}^{2}} \int_{0}^{u}(\gamma y+\bar{\eta}) d y\right)}{\left(u+\frac{\bar{\eta}}{\gamma}\right)^{2}} d u+\frac{h}{\bar{\eta}} W
$$

for some $C>0$ which is determined by the equation $V^{\prime}(\bar{W})=1$. For any $W>0$, we have $V(W)>W$, and $V$ is concave.

Unlike in the previous case the agent is always risk averse, even when $W$ is low. Intuitively, since the incentives are already sufficient to guarantee that the agent exerts effort, additional risk exposure only serves to increase the firing probability for the agent.

\subsection{Drift Ambiguity}

In this section I consider the case where the principal is ambiguity averse about the drift of the output process $\mu$. Just as in the effort cost ambiguity case, I assume that the principal thinks that it is possible for $\mu_{t}$ to follow an $\mathcal{F}_{t}^{B}$ adapted process with $\mu_{t} \in[\underline{\mu}, \bar{\mu}]$ for all $t$.

In the previous case of effort cost ambiguity, the principal could learn the process only from the agent's reports, and I have shown that in equilibrium, the principal can never profit from inducing the agent to reveal his information. Depending on the specification of the principal's preferences, ${ }^{7}$ with drift ambiguity it is possible that the principal learns about $\mu$ even though she is ambiguity averse. For instance if she believes that the drift is $\underline{\mu}$ but observes a long history of relatively high output, she might adjust her belief upward.

\footnotetext{
${ }^{7}$ See for instance Epstein and Schneider (2007).
} 
Since the focus of the present paper is ambiguity aversion, I assume that the principal does not learn from observing the output process, so that her belief about $\mu_{t}$ is determined by ambiguity alone, and does not rely on her observations of $X_{t}{ }^{8}$

As in Section 4 I start with establishing the law of motion for the agent's continuation value. Let $\mu_{P t}$ be the principal's belief about $\mu_{t}$ at time $t$. Since both principal and agent observe $X_{t}$ and they hold different beliefs about the dynamics of the system, their respective filtrations will be different. In other words, $\mathcal{F}_{t}^{A} \neq \mathcal{F}_{t}^{P}$. Under the principal's filtration we have

$$
d X_{t}=\mu_{P t} a_{p t} d t+\sigma d B_{t}^{P}
$$

where $a_{p t}$ is the prescribed effort level that the agent would follow under the contract. Here, I have written $B_{t}^{p}$ to indicate that $B_{t}^{p}$ is a Brownian Motion with respect to the principal's information set. Under the worst-case process $\mu_{P t}$, the agent's continuation value will follow

$$
d W_{t}=\left(\gamma W_{t}+h a_{P t}\right) d t+\psi_{t} d B_{t}^{P}
$$

for some adapted process $\psi_{t}$. As in the previous case, the principal will set

$$
\psi_{t}=\frac{\sigma}{\mu_{P t}} h
$$

whenever $a_{P t}=1$.

Thus, we can establish the analog of Proposition 5 for the principal's value function.

Proposition 11. Suppose the principal does not know the worst-case process $\mu_{t}$ before choosing the contract. The principal's value function is $C^{2}$, satisfies

$$
r J(W)=\max _{\psi} \min _{\mu} \mu a(\psi, \mu)+J^{\prime}(W)(\gamma W+h a(\psi, \mu))+J^{\prime \prime}(W) \frac{1}{2} \psi^{2}
$$

subject to the boundary conditions $J(0)=0$ and $J^{\prime}(\bar{W})=-1$, where

$$
a(\psi, \mu)=\mathbf{1}\left\{\psi \geq \frac{\sigma}{\mu} h\right\}
$$

\footnotetext{
${ }^{8}$ When the principal adjusts her beliefs depending on the history of output observed, the agent has an additional incentive to shirk, since doing so lowers the principal's belief of $\mu$ in the long run. See Giat et al. (2010) for a model based on this premise in a Bayesian setting with disagreement.
} 
and the worst-case prior is $\mu_{t}=\underline{\mu}$ for all $t$ while risk exposure follows

$$
\psi_{t}=\bar{\psi} \equiv \frac{\sigma}{\underline{\mu}} h
$$

for all $t$.

The rationale for the form of the contract is similar to Proposition 5. When the principal sets $\psi<\bar{\psi}$, which is the highest possible risk exposure, nature will be able to induce the agent to shirk by setting a low payoff $\mu$. Thus, we must have that $\psi_{t}=\bar{\psi}$ for all $t$. In this case, although nature cannot make the agent shirk anymore, she can still affect the principal's payoff through her choice of $\mu$. Since the average payoff enters the principal's value function with a positive sign, nature will choose $\mu_{t}=\underline{\mu}$ at all times to minimize the principal's payoff. The reason why the worst-case process is changing over time in the effort ambiguity case, while in the drift ambiguity case it is constant, lies in the way the parameter enters the principal's value function. In the current case, the principal's payoff is always a positive function of $\mu$ at any point in time. $\mu$ also has an indirect effect on the principal's payoff via the risk exposure channel. A lower $\mu$ implies a higher risk exposure to the agent, which in turn increases the probability of termination, and is thus costly for the principal. Since both the direct and indirect effect of $\mu$ point in the same direction, the principal will benefit from a high $\mu$ at any point in time and thus the worst-case prior is constant.

With effort cost ambiguity, the parameter $\eta$ only affects the principal's payoff in two indirect ways. By the risk exposure channel, a higher $\eta$ implies a higher $\psi$ in order to incentivize the agent. As we have seen, this effect is the same over time, and does not depend on the particular worst-case process $\eta$, since the principal will always be forced to set $\psi$ at its highest level. At the same time, $\eta$ affects the principal's value function via the cost of incentives. When $W$ is low, this effect is positive, since it lowers the shutdown probability, while when $W$ is high, it is negative. Thus, whether a high $\eta$ is beneficial or detrimental for the principal changes over time, and so does the worst-case process.

Since the worst-case process is $\mu_{t}$ constant, and at the lowest level, it is intuitive that there is no benefit for the principal from learning the ambiguity process. Even if the principal learned the process, nature would still want to set $\mu$ at the lowest level, because it both lowers the principal's payoff from the agent's effort and raises the required risk exposure. Hence, with drift ambiguity there is no benefit for the principal to learn the ambiguity process in advance.

Proposition 12. Suppose that the principal learns the ambiguity process $\left\{\mu_{t}\right\}$ before choosing the contract. The optimal contract, value function and worst-case process are the same as 
in Proposition 11.

Just as in the case with effort cost ambiguity, we can study the agent's value function, which now will take a particularly simple form. The agent knows that the process $d X_{t}$ follows

$$
d X_{t}=\mu_{t} a_{A t} d t+\sigma d B_{t}^{A}
$$

where $a_{A t}$ is the actual effort exerted by the agent and $B_{t}^{A}$ is a Brownian Motion with respect to the agent's information set. Since the agent knows that the contract of the principal will depend on the variable $W_{t}$, which is the belief the principal holds about the agent's continuation value, he will understand that $W_{t}$ is the relevant state variable for $V\left(W_{t}\right)$, the agent's actual continuation value.

Under $\mathcal{F}_{t}^{A}$, by another application of Girsanov's Theorem, $W_{t}$ follows

$$
\begin{aligned}
d W_{t} & =\left(\gamma W_{t}+h a_{p t}\right) d t+\frac{\sigma}{\underline{\mu}} h d B_{t}^{A} \\
& =\left(\gamma W_{t}+h a_{P t}\right) d t+\frac{\sigma}{\underline{\mu}} h\left(d B_{t}^{P}-\frac{1}{\sigma}\left(\underline{\mu} a_{p t}-\mu_{t} a_{A t}\right)\right) \\
& =\left(\gamma W_{t}+\frac{\mu_{t}}{\underline{\mu}} h a_{A t}\right) d t+\bar{\psi} d B_{t}^{P}
\end{aligned}
$$

We can now characterize the agent's value function and effort policy as a function of $W_{t}$.

Proposition 13. Let

$$
V\left(W_{0}\right)=E \int_{0}^{\tau} e^{-\gamma t}\left(d c_{t}-h a_{t} d t\right)
$$

where $W_{t}$ satisfies the law of motion (40). Then $V$ solves

$$
\gamma V(W)=\max _{a}-h a+V^{\prime}(W)\left(\gamma W_{t}+\frac{\mu_{t}}{\underline{\mu}} h a\right)+V^{\prime \prime}(W) \frac{1}{2} \bar{\psi}^{2}
$$

with the boundary conditions $V(0)=0$ and $V^{\prime}(\bar{W})=1$ and the agent always puts in effort. The value function is given by

$$
V(W)=C\left(W+\frac{1}{\gamma} \underline{\mu} \underline{\underline{\mu}} h\right) \int_{0}^{W} \frac{\exp \left(-\frac{1}{\frac{1}{2}\left(\frac{\sigma}{\underline{\underline{\mu}}}\right)^{2}} \int_{0}^{u}\left(\gamma y+\frac{\underline{\mu}}{\underline{\mu}} h\right) d y\right)}{\left(u+\frac{1}{\gamma} \underline{\underline{\mu}} h\right)^{2}} d u+\frac{\mu}{\mu} W
$$


for some strictly positive constant $C$ and is concave everywhere.

The reason why the agent always works is very similar to the over-compensating regime in the effort cost ambiguity case. Since $\mu_{t}=\underline{\mu}$, an agent who works has a drift in $W$ of

$$
\gamma W+\frac{\mu}{\underline{\mu}} h
$$

which is strictly greater than $\gamma W+h$, the drift without ambiguity. Hence, he receives more compensation than necessary for his effort. At the same time $\bar{\psi}$ is greater than $\frac{\sigma}{\mu} h$, which is the level of risk exposure without ambiguity. Thus, for all $W$ the agent is overcompensated and over-incentivized. Formally, we can show that if the agent always works, we have $V(W) \geq W$ for all $W$, which is analog to the case where $h$ was sufficiently low under effort cost ambiguity.

\section{Conclusion}

In a simple static contracting environment, ambiguity aversion on the principal's part is prone to lead to a trivial characterization of ambiguity and its impact on the optimal contract. ${ }^{9}$ A contribution of my work is showing that in dynamic environments, even when the payoff and utility functions are relatively simple, the worst-case process is not trivial. In particular, it is not obtained by simply setting the parameter which is ambiguous to the worst-case realization from the static problem at all times, and it is possible to obtain meaningful dynamics of the worst-case.

When the ambiguity is about the agent's effort cost, the worst-case realization at each time $t$ will depend on how close the agent is to termination. When the likelihood of termination in the future is relatively high, the worst-case is a low effort cost, since the principal would benefit from raising the agent's continuation value, which translates into a decrease in the firing probability. On the other hand, when termination is relatively unlikely, the worst case features a high effort cost, since then the principal will have to pay the agent high compensation for the effort demanded. This gives rise to two regions, which we can interpret as over- and under-compensation. Both these regions exhibit persistence and the agent can move between them only after a sufficiently high or low history of performance.

The second contribution of my paper is to study the agent's incentives under a misspecified

\footnotetext{
${ }^{9}$ Bergemann and Schlag (2011) provide a specific example for this phenomenon, and the reader may think of other setups where the impact of ambiguity will be straightforward.
} 
contract. In the real world, we should not expect that firms have precise knowledge about how much disutility a manager associates with working on a particular task, or whether the firm's business environment makes it easy or difficult for the manager to fulfill is duties. When the agent's effort cost cannot be credibly communicated, the agent's contract will not be tailored to his particular effort cost, and the agent's incentives may be not sufficient to always motivate him to work. As shown in Section 5, this happens exactly when firing is relatively likely and the agent's true effort cost is high. Thus, firing policies may have a detrimental effect on the agent's incentives at the bottom of the hierarchy, and some agents will shirk even though they know that doing so will lead them to be fired in expectation.

Incentives at the top on the other hand are always sufficient to generate effort, although they too will be decreasing the close the agent is to receiving his payouts. This effect however, is never strong enough to compromise the agent's desire to work. Consequently, unless the agent's effort cost is very low, his incentives to work are strongest in the middle, when the agent is still far away from being paid, and firing is a possibility.

Finally, as sections 6.1 and 6.2 show, obtaining meaningful dynamics for the contract, or the worst-case depends on the actual specification of what is ambiguous. Hence, ambiguity will only exhibit dynamics if the weight the principal attaches to the parameter in question changes its sign over time, and it will remain at a constant value otherwise. 


\section{A Proofs}

Lemma 14. If there exists a number $M$ such that if $\bar{\eta}>M$ then the only contract implemented specifies $a_{t}=0$ everywhere.

Proof. We have that for all $\eta$, and contracts $\alpha$, the principal's payoff is smaller than the first best payoff under $\eta$. Without incentive compatibility the contract for the agent can be taken to be deterministic wlog. Because of the agent's impatience there is a lump sum payment $C_{0}$ to the agent when the contract starts to that

$$
W_{0}=C_{0}-\int_{0}^{\infty} e^{-\gamma t} \eta_{t}^{\alpha} a_{t} d t
$$

and the principal's first best payoff is given by

$$
\begin{aligned}
J_{0}^{f b} & =\sup _{\left\{a_{t}\right\}} \inf _{\left\{\eta_{t}\right\}} \int_{0}^{\infty} e^{-r t} \mu a_{t}-C_{0} \\
& =\sup _{\left\{a_{t}\right\}} \inf _{\left\{\eta_{t}\right\}} \int_{0}^{\infty}\left(e^{-r t} \mu-e^{-\gamma t} \eta_{t}^{\alpha}\right) a_{t} d t-W_{0}
\end{aligned}
$$

where we have the restriction $\eta_{t} \in[\underline{\eta}, \bar{\eta}]$ for all $t$. Now if $a_{t} \neq 0$ for any set $\mathcal{T} \subset \mathbb{R}_{+}$of positive Lebesgue measure, then if $\bar{\eta}$ is high enough we can drive the principal's profit below zero. Thus, $a_{t}=0$ almost everywhere and since effort decisions which are equivalent up to measure zero yield the same payoff we take $a_{t}=0$ everywhere wlog.

The proof for Proposition 5 is below.

Proof. Given the martingale representation in equation (7), the agent will exert effort only if $\psi \geq \frac{\sigma}{\mu} \eta$.

Conjecture that $J^{\prime \prime}<0$ everywhere. The problem can be understood as nature and the principal playing a game, with the latter acting as a Stackelberg leader.

For any $\psi$, nature's problem is

$$
\inf _{\eta \in[\underline{\eta}, \bar{\eta}]}\left(\mu+J^{\prime} \eta\right) a(\psi, \eta)
$$

Since $J^{\prime} \geq-1$ and $\mu>\bar{\eta}$, nature will always prefer that $a(\psi, \eta)=0$. Thus, whenever $\psi<\bar{\psi}$, 
nature will want to set $\eta>\psi \frac{\mu}{\sigma}$ and

$$
\left(\psi \frac{\mu}{\sigma}, \bar{\eta}\right]=\arg \inf _{\eta \in[\underline{\eta}, \bar{\eta}]}\left(\mu+J^{\prime} \eta\right) a(\psi, \eta)
$$

If $\psi=\bar{\psi}$, nature can never induce $a=0$. If $J^{\prime}<0$ it will choose $\eta=\bar{\eta}$ while if $J^{\prime}>0$ it will choose $\eta=\underline{\eta}$.

Since the principal wants to induce $a=1$, and knows that $a=0$ whenever $\psi<\bar{\psi}$, she will choose $\psi=\bar{\psi}$ always.

Given this policy, the HJB equation becomes

$$
r J(W)=\mu+J^{\prime}(W)(\gamma W+\eta(W))+J^{\prime \prime}(W) \frac{1}{2} \bar{\psi}^{2}
$$

where $\eta(W)=\bar{\eta}$ if $J^{\prime} \leq 0$ and $\eta(W)=\underline{\eta}$ otherwise.

Consider the operator

$$
H(W, V, P, M)=-r V+\mu+P(\gamma W+\eta(W))+\frac{1}{2} M \bar{\psi}^{2}
$$

which is a Lipschitz continuous function in $V, P$ and $M$ and measurable in $W$, and thus a Caratheodory function. Consider the solution of the HJB equation subject to the boundary conditions $J(0)=0$ and $J^{\prime}(\bar{W})=-1$.

By Strulovici and Szydlowski (2011), the problem has a unique solution which satisfies the HJB equation almost everywhere and is $C^{1}$ everywhere and has a second derivative almost everywhere.

For each process $\left\{\psi_{t}\right\}$, let $\eta(\psi)$ be adapted process defined by

$$
\eta_{t}(\psi) \in \inf _{\eta_{t}} \mu a\left(\psi_{t}, \eta_{t}\right)+J^{\prime}\left(W_{t}\right)\left(\gamma W_{t}+\eta_{t} a\left(\psi_{t}, \eta_{t}\right)\right)+\frac{1}{2} J^{\prime \prime}\left(W_{t}\right) \psi_{t}^{2} a\left(\psi_{t}, \eta_{t}\right)
$$

We have for all pairs $\left(\tilde{\psi}_{t}, \eta_{t}(\tilde{\psi})\right)$ for which the contract satisfies the IR conditions and implements effort

$$
r J\left(W_{t}\right) \geq \inf _{\eta_{t}} \mu+J^{\prime}\left(W_{t}\right)\left(\gamma W_{t}+\eta_{t}\right)+J^{\prime \prime}\left(W_{t}\right) \frac{1}{2} \tilde{\psi}_{t}^{2}
$$

Let $\tau_{c}$ be the first hitting time at which the agent receives a positive payout and $\tau$ the time 
he gets fired, and let $\kappa=t \wedge \tau \wedge \tau_{c}$ where $a \wedge b=\min \{a, b\}$. Since $J \in C^{1}$ and $J^{\prime \prime}$ exists almost everywhere, we can apply Dynkin's formula, so that

$E e^{-r \kappa} J\left(W_{t}\right)-J\left(W_{0}\right)=E \int_{0}^{\kappa} e^{-r t}\left(-r J\left(W_{t}\right)+J^{\prime}\left(W_{t}\right)\left(\gamma W_{t}+\tilde{\eta} a\left(\tilde{\psi}_{t}, \tilde{\eta}_{t}\right)\right)+\frac{1}{2} J^{\prime \prime}\left(W_{t}\right) \tilde{\psi}_{t}^{2} a\left(\tilde{\psi}_{t}, \tilde{\eta}_{t}\right)\right)$

Then we have

$$
\begin{aligned}
J\left(W_{0}\right) & \geq E\left(\int_{0}^{\kappa} e^{-r t} \mu d t+e^{-r \kappa} J\left(W_{\kappa}\right)\right) \\
& =\inf _{\eta \in H} E\left(\int_{0}^{\kappa} e^{-r t} \mu \tilde{a}_{t} d t+e^{-r \kappa} J\left(W_{\kappa}\right)\right)
\end{aligned}
$$

Since $\tilde{\psi}$ was arbitrary we have from the above that $J$ is greater or equal than the actual value function.

By a similar argument, for all $\tilde{\eta}_{t}$

$$
r J\left(W_{t}\right) \leq \sup _{a} \mu+J^{\prime}\left(W_{t}\right)\left(\gamma W_{t}+\tilde{\eta}_{t}\right)+\frac{1}{2} J^{\prime \prime}\left(W_{t}\right) \tilde{\psi}_{t}^{2}
$$

and we get that

$$
J\left(W_{0}\right) \leq \sup _{a} E\left(\int_{0}^{\kappa} e^{-r t} \mu a_{t} d t+e^{-r \kappa} J\left(W_{\kappa}^{P}\right)\right)
$$

so that $J$ is smaller or equal than the actual value function. Combining the two arguments we see that $J$ must be the actual value function.

To obtain the concavity of $J$, I employ a series of transformations which allow to reduce the HJB equation under effort to study a simpler equation, which allows to draw conclusions from $J$. First, write the HJB equation under effort as

$$
J^{\prime \prime}\left(W_{t}\right)=\frac{r J\left(W_{t}\right)-\mu-\left(\gamma W_{t}+\eta_{t}\right) J^{\prime}\left(W_{t}\right)}{\frac{1}{2} \bar{\psi}^{2}}
$$

Let $\tilde{J}=J-\frac{\mu}{r}$ and notice that $\tilde{J}<0$ for all $W$, otherwise $J$ would attain the first best. Then, $\tilde{J}$ satisfies a version of the HJB equation,

$$
\tilde{J}^{\prime \prime}(W)=\frac{r \tilde{J}(W)-(\gamma W+\eta(W)) \tilde{J}^{\prime}(W)}{\frac{1}{2} \bar{\psi}^{2}}
$$


or equivalently

$$
\tilde{J}^{\prime \prime}(W)=\kappa_{0}(W) \tilde{J}(W)+\kappa_{1}(W) \tilde{J}^{\prime}(W)
$$

with $\kappa_{0}>0$. For a further transformation define the function $\lambda(W)$ by

$$
\lambda(W)=\int_{0}^{W} \exp \left(\int_{0}^{x} \kappa_{1}(y) d y\right) d x
$$

and note $\lambda \geq 0$ and $\lambda^{\prime}(W)=\exp \left(\int_{0}^{W} \kappa_{1}(x) d x\right)>0$.

Consider the change of variables, $V=\lambda(W)$ and $\hat{J}(V)=\tilde{J}\left(\lambda^{-1}(V)\right)$. Then

$$
\hat{J}^{\prime \prime}(V)=\left(\lambda^{\prime}\left(\lambda^{-1}(V)\right)\right)^{-2} \kappa_{0}\left(\lambda^{-1}(V)\right) \tilde{J}^{\prime \prime}\left(\lambda^{-1}(V)\right)
$$

and since $\kappa_{0} \geq 0, \tilde{J}^{\prime \prime}$ and $\hat{J}^{\prime \prime}$ share the same sign. Moreover we have $\hat{J}^{\prime}(V)>0$ whenever $\tilde{J}^{\prime}\left(\lambda^{-1}(V)\right)>0$.

By taking derivatives it can be shown can see that if $\tilde{J}$ satisfies (56) then $\tilde{J}$ satisfies

$$
\hat{J}^{\prime \prime}(V)=\hat{\kappa}(V) \hat{J}(V)
$$

From (58) we can see that if for some $V \hat{J}(V)<0$ and $\hat{J}^{\prime}(V)<0$ then by concavity $\hat{J}^{\prime}\left(V^{\prime}\right)<0$ for all $V^{\prime}>V$ and if $\hat{J}^{\prime}(V)>0$ then $\hat{J}^{\prime}\left(V^{\prime}\right)>0$ for all $V^{\prime}<V$, that is $\hat{J}^{\prime}$ crosses zero at most once. By the construction of $\hat{J}$ from $\tilde{J}$ we see that $J^{\prime}$ crosses zero at most once. Further, if $\tilde{J}$ has a zero for some $W$ then $\tilde{J}^{\prime}$ cannot have a zero. Note that the solution to (56) is unique and that $\tilde{J}(W)=0$ is a solution.

If $J^{\prime} \geq 0$ then we have $J^{\prime \prime}<0$, thus $J^{\prime \prime} \geq 0$ only if $J^{\prime}<0$. Since $J^{\prime} \geq-1$ for all $W$ and $J^{\prime}(\bar{W})=-1$ we must have $J^{\prime \prime}<0$ on $(\bar{W}-\varepsilon, \bar{W})$ for some $\varepsilon>0$.

Consider $W_{0}=\sup \left\{W: J^{\prime \prime}(W)=0\right\}$. Then, we have at $W_{0}$ by the HJB equation

$$
r J\left(W_{0}\right)=\mu+\left(\gamma W_{0}+\bar{\eta}\right) J^{\prime}\left(W_{0}\right)
$$

Note that at $W_{0}$ we have $J^{\prime}<0$ and because $J \in C^{1}$ everywhere, there exists a neighborhood on which $\eta=\bar{\eta}$. On this neighborhood, $J$ is twice continuously differentiable, hence

$$
r J^{\prime}\left(W_{0}\right)=\gamma J^{\prime}\left(W_{0}\right)+\left(\gamma W_{0}+\bar{\eta}\right) J^{\prime \prime}\left(W_{0}\right)
$$


which implies that $J^{\prime}\left(W_{0}\right)=0$ since $\gamma \neq r$. But then the HJB equation implies that $J\left(W_{0}\right)=\frac{\mu}{r}$ or equivalently $\tilde{J}\left(W_{0}\right)=0$ and $\tilde{J}\left(W_{0}\right)$ and $\tilde{J}^{\prime}\left(W_{0}\right)$ have a zero at the same point, which is a contradiction. Thus, it must be the case that $J^{\prime \prime}<0$ everywhere.

\section{A.1 Proof of Proposition (5)}

Proof. Since $a$ is binary and $\eta \in[\underline{\eta}, \bar{\eta}]$, we have that the operator $H: \mathbb{R}_{+} \times \mathbb{R}_{+} \times \mathbb{R} \times \mathbb{R} \rightarrow \mathbb{R}$ given by

$$
H(W, V, P, M)=-r V+\sup _{a} \inf _{\eta} \mu a+P(\gamma W+\eta a)+\frac{1}{2} M \frac{\sigma^{2}}{\mu^{2}} \eta^{2} a
$$

is Lipschitz continuous everywhere in all its variables. By Hille (1968), there exists a $C^{2}$ solution to the HJB equation. For each $\alpha$, let $\eta(\alpha)$ be adapted process defined by

$$
\eta_{t}(\alpha) \in \inf _{\eta_{t}} \mu a_{t}+J^{\prime}\left(W_{t}\right)\left(\gamma W_{t}+\eta_{t} a_{t}\right)+\frac{1}{2} J^{\prime \prime}\left(W_{t}\right) \frac{\sigma^{2}}{\mu^{2}} \eta_{t}^{2} a_{t}
$$

We have for all $\tilde{\alpha}$ such that given the pair $(\tilde{\alpha}, \eta(\tilde{\alpha}))$ the contract satisfies the agent's IC and IR conditions

$$
r J\left(W_{t}\right) \geq \inf _{\eta_{t}} \mu \tilde{a}_{t}+J^{\prime}\left(W_{t}\right)\left(\gamma W_{t}+\eta_{t} \tilde{a}_{t}\right)+\frac{1}{2} J^{\prime \prime}\left(W_{t}\right) \frac{\sigma^{2}}{\mu^{2}} \eta_{t}^{2} \tilde{a}_{t}
$$

Let $\tau_{c}$ be the first hitting time at which the agent receives a positive payout and $\tau$ the time he gets fired, and let $\kappa=t \wedge \tau \wedge \tau_{c}$ where $a \wedge b=\min \{a, b\}$. By Dynkin's Formula,

$$
E e^{-r \kappa} J\left(W_{t}\right)-J\left(W_{0}\right)=E \int_{0}^{\kappa} e^{-r t}\left(-r J\left(W_{t}\right)+J^{\prime}\left(W_{t}\right)\left(\gamma W_{t}+\eta_{t}(\tilde{a}) \tilde{a}_{t}\right)+\frac{1}{2} J^{\prime \prime}\left(W_{t}\right) \frac{\sigma^{2}}{\mu^{2}} \eta(\tilde{a})^{2} \tilde{a_{t}}\right) d t
$$

and hence

$$
\begin{aligned}
J\left(W_{0}\right) & \geq E_{\eta(\tilde{\alpha})}\left(\int_{0}^{\kappa} e^{-r t} \mu \tilde{a}_{t} d t+e^{-r \kappa} J\left(W_{\kappa}\right)\right) \\
& =\inf _{\eta} E_{\eta}\left(\int_{0}^{\kappa} e^{-r t} \mu \tilde{a}_{t} d t+e^{-r \kappa} J\left(W_{\kappa}\right)\right)
\end{aligned}
$$

Since $\tilde{a}$ was arbitrary we have from the above that $J$ is greater or equal than the actual value function. 
By a similar argument, for all $\tilde{\eta}_{t}$

$$
r J\left(W_{t}\right) \leq \sup _{a} \mu a+J^{\prime}\left(W_{t}\right)\left(\gamma W_{t}+\tilde{\eta}_{t} a\right)+\frac{1}{2} J^{\prime \prime}\left(W_{t}\right) \frac{\sigma^{2}}{\mu^{2}} \tilde{\eta}_{t}^{2} a
$$

and we get that

$$
J\left(W_{0}\right) \leq \sup _{a} E_{\tilde{\eta}}\left(\int_{0}^{\kappa} e^{-r t} \mu a_{t} d t+e^{-r \kappa} J\left(W_{\kappa}^{P}\right)\right)
$$

so that $J$ is smaller or equal than the actual value function. Combining the two arguments we see that $J$ must be the actual value function.

\section{A.2 Agent's Value Function}

Proposition 15. The agent's HJB equation (25) has a unique solution which solves the equation for all points in $[0, \bar{W}] \backslash W_{0}$, is $C^{1}$ everywhere on $[0, \bar{W}]$ and $C^{2}$ on $[0, \bar{W}] \backslash W_{0}$. This solution is bounded everywhere and has bounded first derivatives and equals the agent's value function almost everywhere.

Proof. The operator

$$
H(W, V, p, M)=\max _{a}-h a+p \cdot(\gamma W+(\eta(W)-\bar{\eta})+\bar{\eta} a)+M \cdot \frac{1}{2} \bar{\psi}^{2}
$$

is a Lipschitz continuous function, in $V, p$ and $M$ and measurable in $W$. Thus the HJB equation (25) admits a bounded $C^{1}$ solution on $(0, \bar{W})$. The argument is analogous to the one in Proposition 5. First, note that the solution to equation (25) must also be the value function. This result follows from a version of the Feynman-Kac theorem.

Take $F\left(W_{t}\right)=e^{-\gamma t} V\left(W_{t}\right)$ and let $a(W)$ be the optimal effort choice of the agent in equation (25). By Ito's Lemma for semimartingales,

$$
\begin{aligned}
d F= & e^{-\gamma t}\left(-\gamma V\left(W_{t}\right)+\left(\gamma W_{t}+(\eta(W)-\bar{\eta})+\bar{\eta} a\left(W_{t}\right)\right) V^{\prime}\left(W_{t}\right)+V^{\prime \prime}\left(W_{t}\right) \frac{1}{2} \bar{\psi}^{2}\right) d t \\
& +e^{-\gamma t} V^{\prime}\left(W_{t}\right)\left(-d c_{t}+\bar{\psi} a^{P}\left(W_{t}\right) d B_{t}\right) \\
= & e^{-\gamma t}\left(h a\left(W_{t}\right) d t+V^{\prime}\left(W_{t}\right)\left(-d c_{t}+\bar{\psi} d B_{t}\right)\right)
\end{aligned}
$$

Remember that $\tau$ is the firing time and satisfies $\tau=\inf \left\{t: W_{t}=0\right\}$. We have for some 
constant $T>0$

$$
\begin{aligned}
E\left(F\left(W_{\tau \wedge T}\right)-F\left(W_{0}\right)\right) & =E e^{-\gamma(\tau \wedge T)} V\left(W_{\tau \wedge T}\right)-V\left(W_{0}\right) \\
& =E\left(\int_{0}^{\tau \wedge T} e^{-\gamma t}\left(h a\left(W_{t}\right)-V^{\prime}\left(W_{t}\right) d c_{t}\right)\right)
\end{aligned}
$$

Note that $d c_{t}=0$ whenever $W \neq \bar{W}$ and therefore we can replace $V^{\prime}\left(W_{t}\right) d c_{t}$ with $V^{\prime}(\bar{W}) d c_{t}$ so that

$$
V\left(W_{0}\right)=E\left(\int_{0}^{\tau \wedge T} e^{-\gamma t}\left(V^{\prime}\left(\bar{W}_{p}\right) d c_{t}-h a_{t}\right)\right)+E e^{-\gamma(\tau \wedge T)} V\left(W_{\tau \wedge T}\right)
$$

By boundedness of $V$,

$$
\lim _{T \rightarrow \infty} E e^{-\gamma T} V\left(W_{T}\right)=0
$$

Therefore, noting that $W_{\tau}=0$, setting the boundary conditions to $V(0)=0$ and $V^{\prime}\left(\bar{W}_{p}\right)=$ 1 we see that the solution to (25) also satisfies (18).

Since the solution to the agent's HJB equation is unique, whenever a pair of value function and effort policy is found which satisfies the HJB equation and the conditions for exerting effort, it must be the optimal value function. In the following, I construct different solutions to the HJB equation depending on the agent's effort cost $h$.

Lemma 16. For any $n \in \mathbb{R}$ let

$$
I_{n}(W)=\int_{0}^{W} \frac{\exp \left(-\frac{1}{\frac{1}{2} \bar{\psi}^{2}} \int_{0}^{u}(\gamma y+n) d y\right)}{\left(u+\frac{n}{\gamma}\right)^{2}} d u
$$

On any interval $\left[W_{1}, W_{2}\right] \subset\left[0, W_{0}\right]$ on which the agent shirks, the solution to the HJB equation on $\left[W_{1}, W_{2}\right]$ satisfies

$$
V_{l s}(W)=\left(W-\frac{\bar{\eta}-\underline{\eta}}{\gamma}\right)\left(C_{l s 1}+C_{l s 2} I_{\underline{\eta}-\bar{\eta}}(W)\right)+\frac{h}{\bar{\eta}} W-\frac{h}{\gamma} \frac{\bar{\eta}-\underline{\eta}}{\bar{\eta}}
$$

If the agent works on $\left[W_{1}, W_{2}\right]$, the solution satisfies

$$
V_{l w}=\left(W+\frac{\underline{\eta}}{\gamma}\right)\left(C_{l w 1}+C_{l w 2} I_{\underline{\eta}}(W)\right)+\frac{h}{\bar{\eta}} W-\frac{h}{\gamma} \frac{\bar{\eta}-\underline{\eta}}{\bar{\eta}}
$$


On any interval $\left[W_{1}, W_{2}\right] \subset\left[W_{0}, \bar{W}\right]$ on which the agent shirks we have

$$
V_{h s}(W)=W\left(C_{h s 1}+C_{h s 2} I_{0}(W)\right)+\frac{h}{\bar{\eta}} W
$$

and if the agent works

$$
V_{h w}(W)=\left(W+\frac{\bar{\eta}}{\gamma}\right)\left(C_{h w 1}+C_{h w 2} I_{\bar{\eta}}(W)\right)+\frac{h}{\bar{\eta}} W
$$

Further $V$ is either concave or convex on any of the above regions.

Proof. I only provide a proof for the first case, the others are analogous. Consider the transformation $\tilde{V}(W)=V(W)-\frac{h}{\bar{\eta}} W$, which will be useful later. For $W<W_{0}$ and shirking, $V$ solves the equation

$$
\gamma V(W)=V^{\prime}(W)(\gamma W-(\bar{\eta}-\underline{\eta}))+V^{\prime \prime}(W) \frac{1}{2} \bar{\psi}^{2}
$$

and since $\tilde{V}^{\prime}(W)=V^{\prime}(W)-\frac{h}{\bar{\eta}}$ and $\tilde{V}^{\prime \prime}(W)=V^{\prime \prime}(W)$ by construction, $\tilde{V}$ solves

$$
\gamma \tilde{V}(W)=\tilde{V}^{\prime}(W)(\gamma W-(\bar{\eta}-\underline{\eta}))-\frac{h}{\bar{\eta}}(\bar{\eta}-\underline{\eta})+\tilde{V}^{\prime \prime}(W) \frac{1}{2} \bar{\psi}^{2}
$$

On any interval $\left[W_{1}, W_{2}\right]$ with $W_{2}<W_{0}$ a particular solution to the equation homogenous equation

$$
\gamma \tilde{V}(W)=\tilde{V}^{\prime}(W)(\gamma W-(\bar{\eta}-\underline{\eta}))+\tilde{V}^{\prime \prime}(W) \frac{1}{2} \bar{\psi}^{2}
$$

is $\tilde{V}(W)=W-\frac{\bar{\eta}-\underline{\eta}}{\gamma}$ and we can verify that any general solution must be of the form

$$
\tilde{V}(W)=\left(W-\frac{\bar{\eta}-\underline{\eta}}{\gamma}\right)\left(C_{1}+C_{2} I_{\underline{\eta}-\bar{\eta}}(W)\right)-\frac{h}{\gamma} \frac{\bar{\eta}-\underline{\eta}}{\bar{\eta}}
$$

for some constants $C_{1}, C_{2} \in \mathbb{R}$. The form for $V(W)$ follows.

To obtain the concavity/convexity result, we can differentiate twice to get for any $n \in \mathbb{R}$ 
and $W \neq-\frac{n}{\gamma}$

$$
\begin{aligned}
V^{\prime \prime}(W) & =-C_{2} \frac{\frac{\gamma}{\frac{1}{\frac{1}{\psi}} \bar{\psi}^{2}} \exp \left(-\frac{1}{\frac{1}{2} \bar{\psi}^{2}} \int_{0}^{W}(\gamma y+n) d y\right)}{\left(W+\frac{n}{\gamma}\right)^{2}} \\
& \propto-C_{2}
\end{aligned}
$$

Hence whenever $C_{2}>0 \mathrm{~V}$ is concave and if $C_{2}<0 \mathrm{~V}$ is convex. Finally, that $V$ is $C^{2}$ whenever $\eta(W)$ does not jump even though the optimal effort policy might change is a direct consequence of Strulovici and Szydlowski (2011).

In the proof above, I have defined a scaled value function $\tilde{V}(W)=V(W)-\frac{h}{\bar{\eta}} W$. The reason for this is that it is optimal for the agent to exert effort whenever $\tilde{V}^{\prime}(W)>0$, so that it is convenient to study $\tilde{V}$ instead of $V$.

Lemma 17. If the agent works at $W_{0}$, he continues working for all $W>W_{0}$.

Proof. We have $\tilde{V}^{\prime}\left(W_{0}\right)>0$ and since $\tilde{V}$ is $C^{1}$ there exists and interval of positive length $\left[W_{0}, W_{0}+K\right]$ on which it must be optimal to continue exerting effort. Suppose that $W_{0}+K<$ $\bar{W}$. Since $\tilde{V}^{\prime}\left(W_{0}\right)>0$ and $\tilde{V}^{\prime}\left(W_{0}+K\right)=0$ we must have $\tilde{V}^{\prime \prime}(W)<0$ on $\left[W_{0}, W_{0}+K\right]$. But by the super contact condition at $W_{0}+K$ we must have $\tilde{V}^{\prime \prime}<0$ for $W_{0}+K+\varepsilon$ for some $\varepsilon>0$ and the agent will shirk on some interval $\left[W_{0}+K, W_{0}+K^{\prime}\right]$ for $K^{\prime}>K$. Since $\tilde{V}$ is either convex or concave on any interval where the effort choice is constant, it must be the case that once the agent shirks for $W>W_{0}$, he must shirk for all $W^{\prime}>W$, since $\tilde{V}^{\prime \prime}$ will stay negative. But since $\tilde{V}^{\prime}(\bar{W})>0$ this is a contradiction to the boundary condition at $\bar{W}$. Hence, once the agent works at $W_{0}$, he must continue working for all $W>W_{0}$.

Proposition 18. If the agent puts in effort at $W=0$, he puts in effort for all $W$.

Proof. If the agent puts in effort at $W=0$ we must have $\tilde{V}^{\prime}(0)>0$ and since $\tilde{V} \in C^{1}$ there exists an interval of positive length on which the agent continues putting in effort. On this interval, $\tilde{V}$ takes the form

$$
\tilde{V}(W)=\left(W+\frac{\underline{\eta}}{\gamma}\right)\left(C_{l w 1}+C_{l w 2} I_{\underline{\eta}}(W)\right)-\frac{h}{\gamma} \frac{\bar{\eta}-\underline{\eta}}{\bar{\eta}}
$$

The boundary condition at zero is $\tilde{V}(0)=0$ which implies $C_{l w 1}=\frac{h}{\eta} \frac{\bar{\eta}-\underline{\eta}}{\bar{\eta}}>0$. If $C_{l w 2}<0$ $\tilde{V}$ is convex and the agent will continue working until $W_{0}$. From Lemma 17, the agent will 
then continue working for all $W$. If $C_{l w 2}>0$ and $\tilde{V}$ is concave, the agent will either keep working until $W_{0}$, in which case he works for all $W$ or will stop working for some $W<W_{0}$. But notice that for $C_{l w 1}>0$ and $C_{l w 2}>0$ we have

$$
\tilde{V}^{\prime}(W)=C_{l w 1}+C_{l w 2} I_{\underline{\eta}}(W)+C_{l w 2} \frac{\exp \left(-\frac{1}{\frac{1}{2} \bar{\psi}^{2}} \int_{0}^{u}(\gamma y+\underline{\eta}) d y\right)}{\left(W+\frac{\underline{\eta}}{\gamma}\right)}>0
$$

for all $W>0$ and hence there cannot exist a point $W<W_{0}$ for which $\tilde{V}^{\prime}(W)=0$.

Proposition 19. If the agent shirks at 0 , he must stop shirking at some $W_{s}<W_{0}$ and then work forever. The value function is convex on $\left[0, W_{s}\right]$ and always concave on $\left[W_{0}, \bar{W}\right]$.

Proof. If the agent shirks at 0 we have for some interval $[0, W]$

$$
\tilde{V}(W)=\left(W-\frac{\bar{\eta}-\underline{\eta}}{\gamma}\right)\left(C_{l s 1}+C_{l s 2} I_{\underline{\eta}-\bar{\eta}}(W)\right)-\frac{h}{\gamma} \frac{\bar{\eta}-\underline{\eta}}{\bar{\eta}}
$$

and the boundary condition $\tilde{V}^{\prime}(W)=0$ implies $C_{l s 1}=-\frac{h}{\bar{\eta}}$. Note that at the point $W=\frac{\bar{\eta}-\underline{\underline{\eta}}}{\gamma}$, $\tilde{V}$ is bounded and positive, since

$$
I_{\underline{\eta}-\bar{\eta}}(W) \geq \int_{0}^{W} \frac{1}{\left(u-\frac{\bar{\eta}-\underline{\eta}}{\gamma}\right)^{2}} d u
$$

and

$$
I_{\underline{\eta}-\bar{\eta}}(W) \leq M \int_{0}^{W} \frac{1}{\left(u-\frac{\bar{\eta}-\underline{\eta}}{\gamma}\right)^{2}} d u
$$

for some bounded positive number $M$ on $\left[0, \frac{\bar{\eta}-\underline{\eta}}{\gamma}\right]$.

If $C_{l s 2}>0$ we have $\tilde{V}^{\prime \prime}<0$ and the agent shirks at least until $W_{0}$. At $W_{0}$, $\tilde{V}^{\prime \prime}$ is not continuous, but taking left and right derivatives, we have

$$
V_{-}^{\prime \prime}\left(W_{0}\right)=\gamma V\left(W_{0}\right)-V^{\prime}\left(W_{0}\right) \gamma W_{0}+(\bar{\eta}-\underline{\eta}) V^{\prime}\left(W_{0}\right)
$$

and

$$
V_{+}^{\prime \prime}\left(W_{0}\right)=\gamma V\left(W_{0}\right)-V^{\prime}\left(W_{0}\right) \gamma W_{0}
$$

Since $V^{\prime}(W) \geq 0$ we have $\tilde{V}_{+}^{\prime \prime}\left(W_{0}\right)=V_{+}^{\prime \prime}\left(W_{0}\right)<0$ and since the agent will shirk at least 
on some interval $\left[W_{0}, W_{0}+K\right]$ he will shirk for all $W>W_{0}$ which contradicts the boundary condition $\tilde{V}^{\prime}(\bar{W})>0$. Thus, $\tilde{V}$ must be convex as long as the agent shirks.

If the agent starts working at the point $W<W_{0}$ we have $\tilde{V}^{\prime}(W)=0$ and $\tilde{V}^{\prime \prime}(W)>0$. Since $\tilde{V}^{\prime \prime}$ is continuous at $W$, on the region $[W, W+K]$ where the agent works, we must have $\tilde{V}^{\prime \prime}>0$. Hence the agent cannot stop working for $W^{\prime}>W$, and will keep working until $W_{0}$, and by Lemma 17 he will continue working for all $W^{\prime}>W_{0}$.

To rule out that $W>W_{0}$ we need to study the concavity property of $V$ at $\left[W_{0}, \bar{W}\right]$. Note that $V^{\prime \prime}(\bar{W})<0$ is equivalent to

$$
\gamma(V(\bar{W})-\bar{W})<\bar{\eta}-h
$$

since the agent will always exert effort on a small interval $(\bar{W}-K, \bar{W})$ because of the boundary condition $V^{\prime}(\bar{W})=1$. If $V(\bar{W}) \leq \bar{W}$ this condition will be satisfied. By the functional forms in Lemma 16, we know that on $(\bar{W}-K, \bar{W}), V$ must remain concave. By the super contact property on $\left[W_{0}, \bar{W}\right], V$ must remain concave even if the agent shirks on $\left[W_{0}, \bar{W}-K\right]$. But this would imply a contradiction since $V^{\prime}(W)>1$ on $\left(W_{0}, \bar{W}\right)$ by the concavity of $V$. Hence, it must be the case that the agent works on $\left[W_{0}, \bar{W}\right]$ and $V$ is concave there. By the continuity of $V^{\prime}(W)$ and $V^{\prime}\left(W_{0}\right)>1$ we thus have $W_{s}<W_{0}$.

If on the other hand $V(\bar{W})>\bar{W}$ and $V^{\prime \prime}(\bar{W})$ is convex, by an analog argument, $V$ must be convex on $\left[W_{0}, \bar{W}\right]$, and thus $V^{\prime}(W)<1$ on $\left[W_{0}, \bar{W}\right]$ and $W_{s}>W_{0}$. The agent shirks on $\left[W_{0}, W_{s}\right]$ and since in this case $V_{+}^{\prime \prime}\left(W_{0}\right)<V_{-}^{\prime \prime}\left(W_{0}\right), V$ must be convex on $\left[0, W_{0}\right]$. But then $V^{\prime}(W)<1$ on $(0, \bar{W})$ and it cannot be true that $V(\bar{W})>\bar{W}$. Hence, whenever $V(\bar{W})>\bar{W}, V$ must be concave close to $\bar{W}$, and the same argument as before applies.

I thus have established that there are only two possible regimes. Either the agent works at zero, and thus keeps working forever, or he shirks at zero and starts working at some point $W_{s}<W_{0}$ and then keeps working for all points greater than $W$. Which of the regimes will be optimal depends solely on the initial slope of $\tilde{V}$, which in turn will depend on the effort cost $h$.

Proposition 20. There exists a cutoff $\hat{h}$ such that for $h>\hat{h}$ the agent initially shirks and for $h<\hat{h}$ the agent always works.

Proof. Let $V_{h}(W)$ be the value function given effort cost parameter $h$ and $V_{h}(W, a)$ the value function given a certain effort policy $a \equiv\left\{a_{t}\right\}_{t \geq 0}$ with $a_{t}=1$ on a set of positive probability. 
We have for any $h^{\prime}>h$

$$
V_{h^{\prime}}(W, a) \leq V_{h}(W, a)
$$

for any $a$ and the inequality is strict for $W>0$. Let

$$
a_{h} \in \arg \sup _{a} V_{h}(W, a)
$$

Then,

$$
V_{h}(W)=V_{h}\left(W, a_{h}\right)<V_{h^{\prime}}\left(W, a_{h}\right) \leq V_{h^{\prime}}\left(W, a_{h^{\prime}}\right)=V_{h^{\prime}}(W)
$$

for $W>0$. Thus, the value function $V_{h}$ is decreasing in $h$, uniformly for all $W$. Since $V_{h}(0)=V_{h^{\prime}}(0)$ for all $h^{\prime}, h>0$ by the boundary condition at zero, we must have $V_{h}^{\prime}(0)>$ $V_{h^{\prime}}(0)$ for $h^{\prime}>h$.

Consider the case $h=\underline{\eta}$. We have $V_{\underline{\eta}}(W)>W$ for $W>0$ and hence $V^{\prime}(0)>1>\frac{\eta}{\bar{\eta}}$. Hence the only solution for an agent with $h=\underline{\eta}$ is to work forever. Similarly, for $h=\bar{\eta}$, we must have $V_{\bar{\eta}}(W)<W$ for $W>0$. For this agent, we must have $V^{\prime}(0)<1=\frac{h}{\bar{\eta}}$ and hence the agent will shirk initially.

By the existence of types which work and shirk initially and monotone dependence of $V_{h}^{\prime}(0)$ on $h$, the result follows.

\section{A.3 Principal's Value Function When Ambiguity is Revealed Be- fore the Contract}

Proof. The verification proof is analog to Proposition 5. For any choice of $\eta$, the principal will be able to induce effort by setting $\psi \geq \frac{\sigma}{\mu} \eta$. Thus, for any choice of $\eta$, nature knows that $a=1$.

Suppose that $J^{\prime \prime}<0$, then given a choice of nature $\eta$, the principal will select $\psi(\eta)=\frac{\sigma}{\mu} \eta$. Thus, her problem is equivalent to

$$
\min _{\eta} J^{\prime}(W) \eta+J^{\prime \prime}(W) \frac{1}{2}\left(\frac{\sigma}{\mu}\right)^{2} \eta^{2}
$$

Since $J^{\prime \prime}<0$, whenever $J^{\prime}<0$ the optimal solution is $\eta_{t}=\bar{\eta}$. When $J^{\prime}>0$, suppose that

$$
J^{\prime}(W) \underline{\eta}+J^{\prime \prime}(W) \frac{1}{2}\left(\frac{\sigma}{\mu}\right)^{2} \underline{\eta}^{2}<J^{\prime}(W) \bar{\eta}+J^{\prime \prime}(W) \frac{1}{2}\left(\frac{\sigma}{\mu}\right)^{2} \bar{\eta}^{2}
$$


so that $\eta_{t}=\eta$ is the optimal choice. Equivalently

$$
\begin{aligned}
J^{\prime}(W)(\underline{\eta}-\bar{\eta}) & <\frac{1}{2} J^{\prime \prime}(W)\left(\frac{\sigma}{\mu}\right)^{2}\left(\bar{\eta}^{2}-\underline{\eta}^{2}\right) \\
& =\left(\bar{\eta}^{2}-\underline{\eta}^{2}\right) \frac{r J(W)-\mu-J^{\prime}(W)(\gamma W+\underline{\eta})}{\underline{\eta}^{2}}
\end{aligned}
$$

where the second equality follows from the form of the HJB equation when $\eta_{t}=\underline{\eta}$ is optimal. Then

$$
J^{\prime}(W)\left(\underline{\eta}^{3}-\bar{\eta} \underline{\eta}^{2}+\underline{\eta} \underline{\eta}^{2}-\underline{\eta}^{3}\right)<\left(r J(W)-\mu-J^{\prime}(W) \gamma W\right)\left(\bar{\eta}^{2}-\underline{\eta}^{2}\right)
$$

The term multiplying $J^{\prime}$ on the LHS is equal to $\underline{\eta} \bar{\eta}(\bar{\eta}-\underline{\eta})>0$, since we assumed $J^{\prime}>0$ and we have $J(W)<\frac{\mu}{r}$ the RHS must be negative and we have a contradiction. Hence, $\eta_{t}=\bar{\eta}$ must be the worst-case process when $J^{\prime}>0$. Given we have a constant process $\eta_{t}$ and constant risk exposure $\psi_{t}=\frac{\sigma}{\mu} \bar{\eta}$, the functional form for $J$ follows directly from DeMarzo and Sannikov (2006).

\section{A.4 Agent's Value Function When Ambiguity is Revealed Before the Contract}

Proof. The proof is analogous to the one in the previous section. In particular, the arguments about existence and uniqueness of the solution to the HJB equation still apply. Note that the agent's value function in this case must satisfy $V(W) \geq W$ and hence $V^{\prime}(0)>1 \geq \frac{h}{\bar{\eta}}$ which holds for all $h$, and the agent will work initially at $W=0$. The value function has closed form solutions similar to the ones in Lemma 16 and the argument from Proposition 18 can be applied to show that the agent cannot stop working once he works at $W=0$.

From the closed form solution, concavity is immediate and together with the boundary condition $V^{\prime}(\bar{W})=1$ this implies that $V^{\prime}(W)>1$ for all $W<\bar{W}$ and thus $V(W)>W$ for all $W>0$.

\section{A.5 Principal's Value Function under Drift Ambiguity}

Proof. The verification argument for the HJB equation is analog to the one in Proposition

5. Suppose that $J$ is concave. If the principal sets $\psi<\bar{\psi} \equiv \frac{\sigma}{\underline{\mu}} h$ then nature can induce the 
agent to shirk by setting

$$
\mu>\frac{\psi \mu}{\sigma h}
$$

In this case, the principal's payoff sinks by

$$
\mu+J^{\prime}(W) h \geq \mu-h>0
$$

Thus, whenever $\psi<\bar{\psi}$, nature will induce the agent to shirk and the principal will set $\psi=\bar{\psi}$. When this is the case, nature can still affect the principal's payoff by setting $\mu$, although she cannot induce the agent to shirk. As is easily seen, nature will set $\mu=\underline{\mu}$ nonetheless, since the principal benefits from high average output. Thus, we have $\mu_{t}=\mu$ and $\psi_{t}=\bar{\psi}$.

In this case, the HJB equation becomes

$$
r J(W)=\underline{\mu}+J^{\prime}(W)(\gamma W+h)+J^{\prime \prime}(W) \frac{1}{2} \bar{\psi}^{2}
$$

which is a version of the equation studied by DeMarzo and Sannikov (2006), and it follows that it must indeed be concave and satisfy the boundary conditions in the Proposition.

\section{A.6 Learning the Worst-Case Process under Drift Ambiguity}

Proof. The principal's value function will solve the equation

$$
r J(W)=\min _{\mu} \max _{\psi} \mu a(\psi, \mu)+J^{\prime}(W)(\gamma W+h a(\psi, \mu))+J^{\prime \prime}(W) \frac{1}{2} \psi^{2}
$$

subject to the same conditions as in Proposition 11. Given any choice of nature, the principal can induce effort by setting

$$
\psi(\mu)=\frac{\sigma}{\mu} h
$$

Thus, nature's problem is

$$
\min \mu+J^{\prime \prime}(W) \frac{1}{2} \frac{\sigma}{\mu} h
$$

and if $J^{\prime \prime}<0$ we have $\mu_{t}=\bar{\mu}$ for all $t$. The rest of the proof proceeds analogously to Proposition 11. 


\section{A.7 Agent's Value Function under Drift Ambiguity}

Proof. The proof is essentially an application of Feynman-Kac. I show that the solution to the ODE (42) indeed satisfies (41). Take $F\left(W_{t}\right)=e^{-\gamma t} V\left(W_{t}\right)$. By Ito's Lemma for Semimartingales

$$
\begin{aligned}
d F= & e^{-\gamma t}\left(-\gamma V+\left(\gamma W+h a_{p}-\frac{\mu a_{p}-\mu a_{a}}{\sigma} \psi_{p}\right) V^{\prime}+V^{\prime \prime}(W) \frac{1}{2}\left(\frac{\sigma}{\underline{\mu}} h\right)^{2}\right) d t \\
& +e^{-\gamma t} V^{\prime}\left(-d c_{t}+\left(\frac{\sigma}{\underline{\mu}} h\right)^{2} d B_{t}\right) \\
= & e^{-\gamma t}\left(h a_{t} d t+V^{\prime}\left(-d c_{t}+\left(\frac{\sigma}{\underline{\mu}} h\right)^{2} d B_{t}\right)\right)
\end{aligned}
$$

Hence for some $T>0$,

$$
\begin{aligned}
E^{a}\left(F\left(W_{\tau \wedge T}\right)-F\left(W_{0}\right)\right) & =E^{a} e^{-\gamma(\tau \wedge T)} V\left(W_{\tau \wedge T}\right)-V\left(W_{0}\right) \\
& =E^{a}\left(\int_{0}^{\tau \wedge T} e^{-\gamma t}\left(h a_{t}-V^{\prime}\left(W_{t}\right) d c_{t}\right)\right)
\end{aligned}
$$

Note that $d c_{t}=0$ whenever $W \neq \bar{W}_{p}$ and therefore we can replace $V^{\prime}\left(W_{t}\right) d c_{t}$ with $V^{\prime}\left(\bar{W}_{p}\right) d c_{t}$ so that

$$
V\left(W_{0}\right)=E^{a}\left(\int_{0}^{\tau \wedge T} e^{-\gamma t}\left(V^{\prime}\left(\bar{W}_{p}\right) d c_{t}-h a_{t}\right)\right)+E^{a} e^{-\gamma(\tau \wedge T)} V\left(W_{\tau \wedge T}\right)
$$

By transversality,

$$
\lim _{T \rightarrow \infty} E^{a} e^{-\gamma T} V\left(W_{T}\right)=0
$$

Therefore, noting that $W_{\tau}=0$, setting the boundary conditions to $V(0)=0$ and $V^{\prime}\left(\bar{W}_{p}\right)=$ 1 we see that the solution to (42) satisfies (41).

We know that $V(W) \geq V_{e}(W)$, where $V_{e}$ is the agent's value function when he puts in effort at all $t$. In this case, $W_{t}$ follows

$$
d W_{t}=\left(\gamma W_{t}+\frac{\mu}{\underline{\mu}} h\right) d t+\bar{\psi} d B_{t}
$$

Since the drift is greater than $\gamma W_{t}+h$, the drift under the principal's information set, for all $t$, it must be the case that $V_{e} \geq W$. Then, we must have $V(\bar{W}) \geq \bar{W}$ and $V^{\prime}(\bar{W})=1$. Note 
that whenever the agent puts in effort or does not put in effort similar functional forms as in Lemma 16 can be obtained. Thus, $V^{\prime \prime}$ is either positive or negative on any region where the agent shirks or works. Further, since all the parameters in the agent's value function besides effort are continuous, $V$ is $C^{2}$ everywhere. Thus, if the agent shirks initially, we must have that $V$ is convex on that region, and by the $C^{2}$ property, convex everywhere. But then together with the boundary condition $V<W \leq V_{e}$ which is a contradiction. Hence, the only possible solution is one where the agent works forever, and $V$ is concave everywhere. This solution admits the closed form solution

$$
\tilde{V}(W)=C\left(W+\frac{1}{\gamma} \frac{\mu}{\underline{\mu}} h\right) \int_{0}^{W} \frac{\exp \left(-\frac{1}{\frac{1}{2}(\underline{\underline{\mu}} h)^{2}} \int_{0}^{u}\left(\gamma y+\frac{\mu}{\underline{\mu}} h\right) d y\right)}{\left(u+\frac{1}{\gamma} \frac{\mu}{\mu} h\right)^{2}} d u
$$

which can be obtained along similar lines as the solutions in Lemma 16. 


\section{References}

Bebchuk, L. and J. Fried (2006). Pay without performance: The unfulfilled promise of executive compensation. Harvard Univ Pr.

Bergemann, D. and K. Schlag (2008). Pricing without priors. Journal of the European Economic Association 6(2-3), 560-569.

Bergemann, D. and K. Schlag (2011). Robust monopoly pricing. Journal of Economic Theory.

Biais, B., T. Mariotti, J. C. Rochet, and S. Villeneuve (2010). Large risks, limited liability, and dynamic moral hazard. Econometrica 78(1), 73-118.

Bose, S. and A. Daripa (2009). A dynamic mechanism and surplus extraction under ambiguity. Journal of Economic theory 144(5), 2084-2114.

Bose, S. and L. Renou (2011). Mechanism design with ambiguous communication devices.

Chen, Z. and L. Epstein (2002). Ambiguity, risk, and asset returns in continuous time. Econometrica 70(4), 1403-1443.

DeMarzo, P., M. Fishman, Z. He, and N. Wang (2010). Dynamic agency and the q theory of investment.

DeMarzo, P. M. and Y. Sannikov (2006). Optimal security design and dynamic capital structure in a continuous-time agency model. Journal of Finance 61(6), 2681-2724.

Di Tillio, A., N. Kos, and M. Messner (2011). The design of ambiguous mechanisms. work in progress.

Epstein, L. and S. Ji (2011). Ambiguous volatility, possibility and utility in continuous time. Arxiv preprint arXiv:1103.1652.

Epstein, L. and M. Schneider (2007). Learning under ambiguity. The Review of Economic Studies 74(4), 1275.

Fong, Y. and J. Li (2010). Relational contracts, efficiency wages, and employment dynamics. Northwestern University, Kellogg School of Management.

Gabaix, X. and A. Landier (2008). Why has CEO pay increased so much? The Quarterly Journal of Economics 123(1), 49.

Garrett, D. (2009). Foundations for simple menus of contracts in cost" based procurement!

Garrett, D. and A. Pavan (2010a). Dynamic managerial compensation: On the optimality of seniority-based schemes. Technical report, Mimeo. 6 . 
Garrett, D. and A. Pavan (2010b). Managerial turnover in a changing world.

Giat, Y., S. Hackman, and A. Subramanian (2010). Investment under uncertainty, heterogeneous beliefs, and agency conflicts. Review of Financial Studies 23(4), 1360-1404.

Gilboa, I. and D. Schmeidler (1989). Maxmin expected utility with non-unique prior. Journal of mathematical economics 18(2), 141-153.

He, Z. (2009). Optimal executive compensation when firm size follows geometric brownian motion. Review of Financial Studies 22(2), 859.

Hille, E. (1968). Lectures on ordinary differential equations. Addison-Wesley.

Karatzas, I. and S. E. Shreve (1991). Brownian motion and stochastic calculus. Springer.

Murphy, K. (2003). The trouble with stock options. Journal of Economic Perspectives 17, 49-70.

Oyer, P. and S. Schaefer (2011). Personnel economics: hiring and incentives. Handbook of Labor Economics 4, 1769-1823.

Piskorski, T. and A. Tchistyi (2011). Stochastic house appreciation and optimal mortgage lending. Review of Financial Studies 24(5), 1407.

Sannikov, Y. (2008). A continuous-time version of the principal-agent problem. Review of Economic Studies 75(3), 957-984.

Schattler, H. and J. Sung (1993). The first-order approach to the continuous-time principal-agent problem with exponential utility. Journal of Economic Theory 61(2), $331-371$.

Spear, S. and C. Wang (2005). When to fire a CEO: optimal termination in dynamic contracts. Journal of Economic Theory 120(2), 239-256.

Strulovici, B. and M. Szydlowski (2011). On the smoothness of value functions.

Wang, C. (2010). Termination of dynamic contracts in an equilibrium labor market model. Journal of Economic Theory.

Zhu, J. (2011). Sticky incentives and dynamic agency. 